\title{
The Structural and Conformational Properties of Formic Hydrazide (Formylhydarzine) Studied by Microwave Spectroscopy and Quantum Chemical Calculations
}

\author{
Svein Samdal and Harald Møllendal* \\ Department of Chemistry, University of Oslo, P. O. Box 1033 Blindern, NO-0315 Oslo, Norway \\ *E-Mail: harald.mollendal@kjemi.uio.no
}




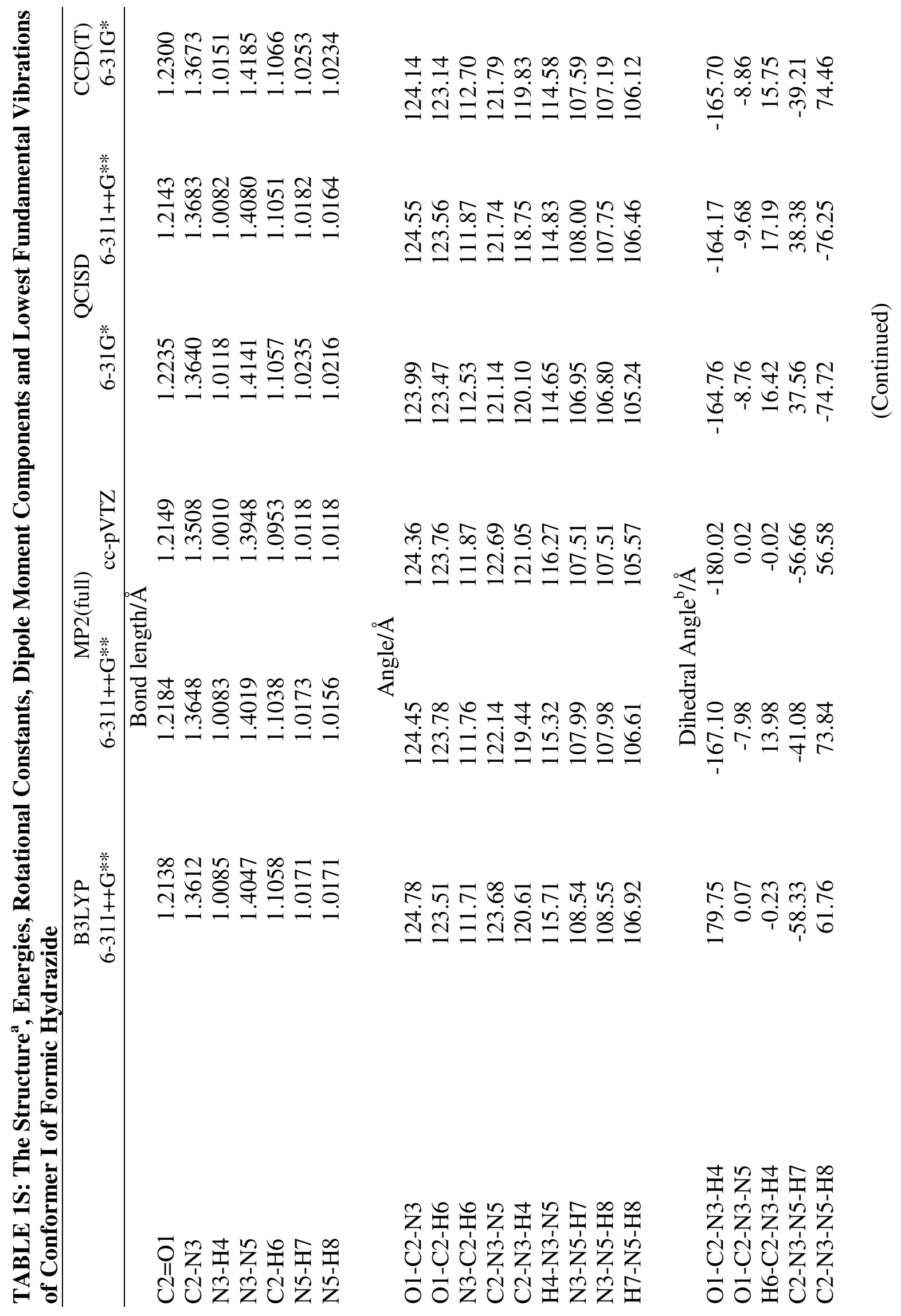




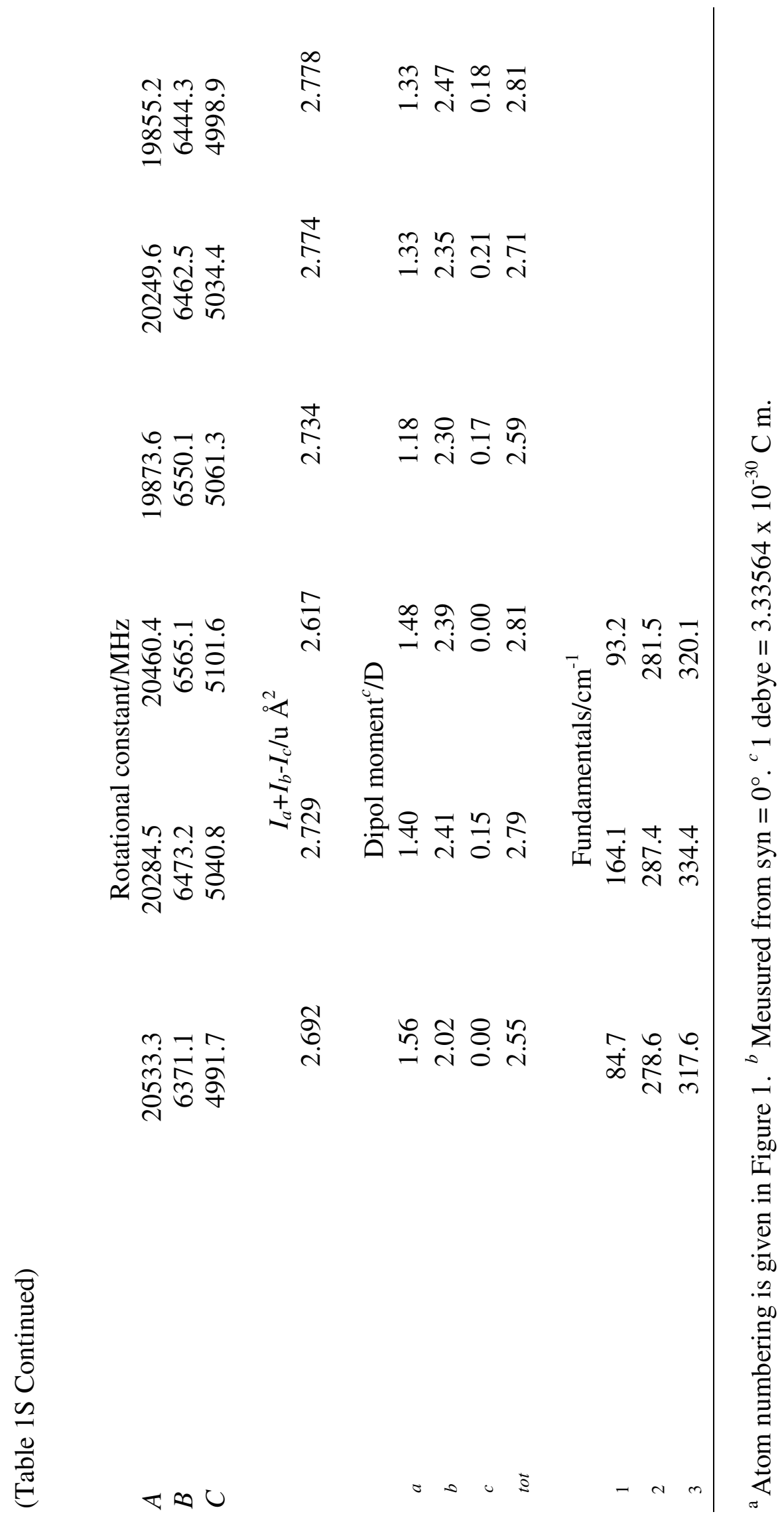




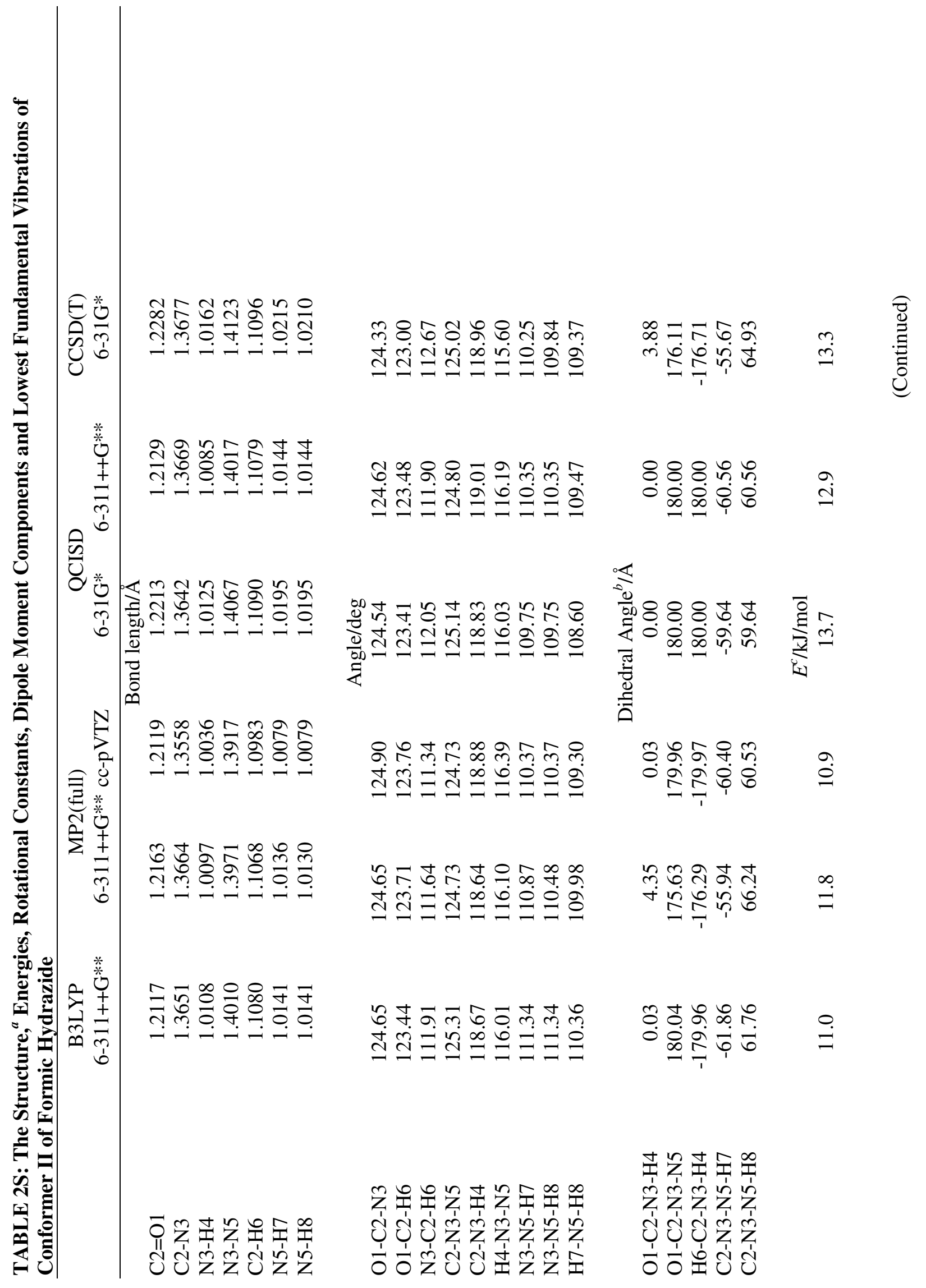




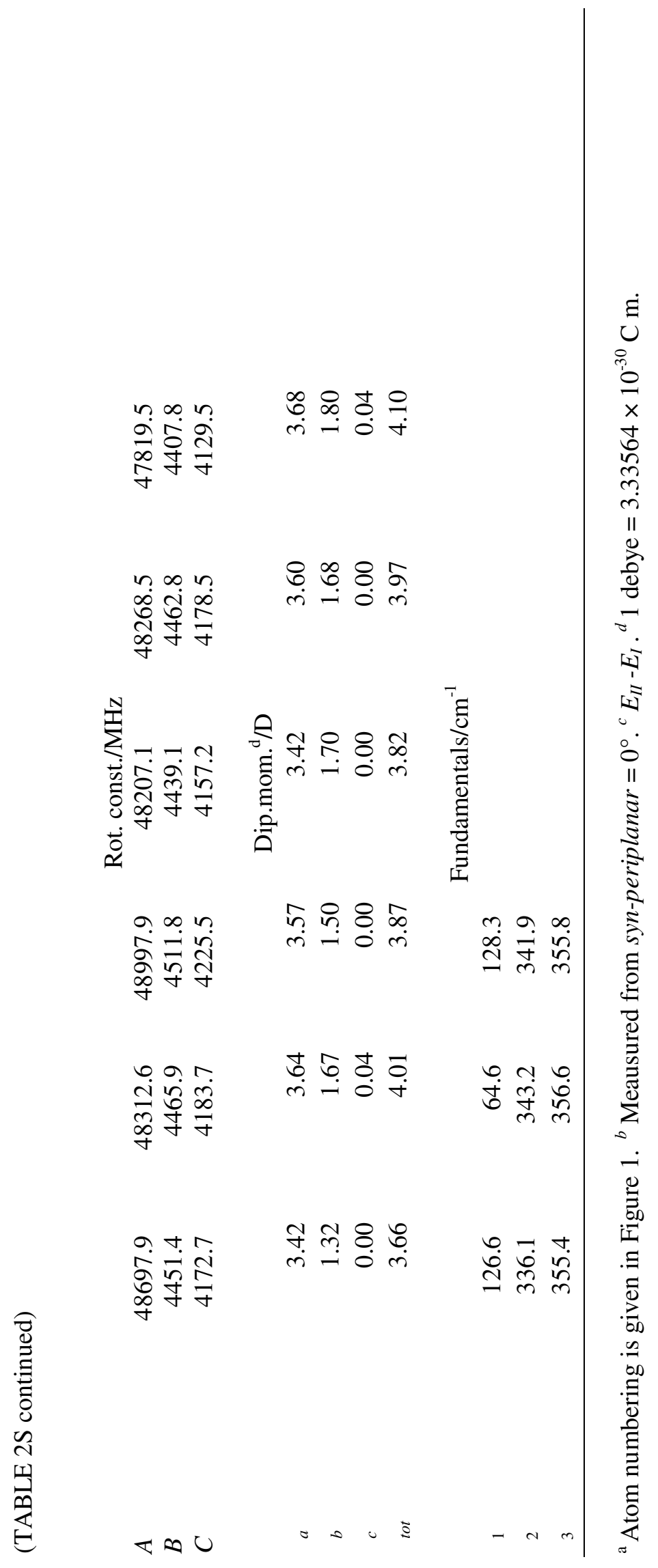


Table 3S: Microwave Spectrum and Spectroscopic Constants of the Ground Vibrational state of Formic Hydrazide

TOTAL NUMBER OF ACCEPTED TRANSITIONS: 278

ACCURACY : $\pm 0.15 \mathrm{MHZ}$

$\mathrm{RMS}=0.1278 \quad$ GRAMDET.$=0.21 \mathrm{E}-46$

TRANSITION OBS.FREQ. O.-C. DISTORTION CONTRIBUTION TOTAL SEXTIC

\begin{tabular}{|c|c|c|c|c|c|c|c|c|c|}
\hline$(\mathrm{J}, \mathrm{K}-$ & $1, \mathrm{~K}$ & $+1-$ & & $\mathrm{K}-1$ & $K+1)$ & FREQ . & DELTA & & \\
\hline 0 & 0 & 0 & 1 & 0 & 1 & 11609.500 & -0.059 & -0.02 & \\
\hline 1 & 0 & 1 & 1 & 1 & 0 & 15065.780 & 0.166 & -0.04 & \\
\hline 0 & 0 & 0 & 1 & 1 & 1 & 25195.170 & -0.260 & -0.05 & \\
\hline 1 & 0 & 1 & 2 & 0 & 2 & 23104.750 & 0.178 & -0.19 & \\
\hline 1 & 1 & 0 & 2 & 1 & 1 & 24698.850 & 0.092 & -0.16 & \\
\hline 2 & 0 & 2 & 2 & 1 & 1 & 16660.070 & 0.270 & -0.02 & \\
\hline 1 & 1 & 1 & 2 & 1 & 2 & 21739.490 & 0.117 & -0.04 & \\
\hline 2 & 1 & 1 & 2 & 2 & 0 & 40871.460 & 0.071 & -0.71 & \\
\hline 2 & 1 & 2 & 2 & 2 & 1 & 45196.140 & 0.027 & -0.85 & \\
\hline 2 & 0 & 2 & 3 & 0 & 3 & 34375.010 & -0.006 & -0.58 & \\
\hline 2 & 1 & 2 & 3 & 0 & 3 & 22154.520 & 0.177 & -0.70 & \\
\hline 2 & 1 & 1 & 3 & 1 & 2 & 36973.070 & -0.105 & -0.72 & \\
\hline 2 & 0 & 2 & 3 & 1 & 3 & 44760.730 & 0.161 & -0.16 & \\
\hline 2 & 1 & 2 & 3 & 1 & 3 & 32540.090 & 0.194 & -0.28 & \\
\hline 2 & 2 & 0 & 3 & 2 & 1 & 35281.790 & 0.054 & -0.16 & -0.01 \\
\hline 3 & 0 & 3 & 4 & 0 & 4 & 45332.040 & 0.038 & -1.23 & -0.01 \\
\hline 3 & 1 & 3 & 4 & 0 & 4 & 34946.580 & 0.131 & -1.65 & \\
\hline 3 & 1 & 2 & 4 & 1 & 3 & 49149.670 & -0.107 & -1.82 & -0.01 \\
\hline 4 & 0 & 4 & 4 & 1 & 3 & 23075.920 & 0.186 & -0.75 & -0.01 \\
\hline 3 & 0 & 3 & 4 & 1 & 4 & 53650.590 & 0.077 & -0.36 & -0.01 \\
\hline 3 & 1 & 3 & 4 & 1 & 4 & 43265.050 & 0.090 & -0.78 & -0.01 \\
\hline 3 & 2 & 1 & 4 & 2 & 2 & 47453.230 & 0.234 & -1.10 & -0.01 \\
\hline 4 & 1 & 3 & 4 & 2 & 2 & 37482.900 & -0.269 & 0.56 & -0.01 \\
\hline 3 & 2 & 2 & 4 & 2 & 3 & 46348.570 & 0.155 & -0.80 & -0.01 \\
\hline 4 & 1 & 4 & 4 & 2 & 3 & 50568.480 & 0.162 & -0.69 & -0.01 \\
\hline 3 & 3 & 0 & 4 & 3 & 1 & 46684.760 & 0.153 & 0.03 & -0.03 \\
\hline 5 & 3 & 2 & 4 & 4 & 1 & 41538.230 & 0.118 & -9.46 & \\
\hline 4 & 0 & 4 & 5 & 0 & 5 & 55938.790 & 0.017 & -2.10 & -0.01 \\
\hline 4 & 1 & 4 & 5 & 0 & 5 & 47620.370 & 0.107 & -2.97 & -0.01 \\
\hline 4 & 1 & 3 & 5 & 1 & 4 & 61182.680 & -0.164 & -3.57 & -0.02 \\
\hline 4 & 1 & 3 & 5 & 1 & 4 & 61182.800 & -0.044 & -3.57 & -0.02 \\
\hline 4 & 2 & 3 & 5 & 1 & 4 & 25371.960 & 0.211 & -4.50 & -0.01 \\
\hline 5 & 0 & 5 & 5 & 1 & 4 & 28319.860 & 0.055 & -2.22 & -0.01 \\
\hline 4 & 2 & 2 & 5 & 2 & 3 & 59896.020 & -0.136 & -2.91 & -0.03 \\
\hline 5 & 1 & 4 & 5 & 2 & 3 & 36196.580 & 0.099 & 1.22 & -0.02 \\
\hline 4 & 2 & 3 & 5 & 2 & 4 & 57791.670 & -0.109 & -2.03 & -0.03 \\
\hline 5 & 1 & 5 & 5 & 2 & 4 & 54458.930 & 0.017 & -1.15 & -0.03 \\
\hline 4 & 3 & 1 & 5 & 3 & 2 & 58500.000 & -0.052 & -1.20 & -0.06 \\
\hline 4 & 4 & 1 & 5 & 4 & 2 & 58302.640 & 0.130 & 0.49 & -0.11 \\
\hline 5 & 1 & 5 & 6 & 0 & 6 & 59954.360 & -0.007 & -4.55 & -0.03 \\
\hline 5 & 1 & 5 & 6 & 0 & 6 & 59954.360 & -0.007 & -4.55 & -0.03 \\
\hline 6 & 0 & 6 & 6 & 1 & 5 & 35100.930 & 0.066 & -5.09 & -0.02 \\
\hline
\end{tabular}




\begin{tabular}{|c|c|c|c|c|c|c|c|c|c|}
\hline 6 & 1 & 6 & 6 & 1 & 5 & 30611.740 & -0.006 & -6.94 & -0.01 \\
\hline 6 & 1 & 5 & 6 & 2 & 4 & 35730.090 & -0.072 & 1.37 & -0.05 \\
\hline 6 & 1 & 6 & 6 & 2 & 5 & 59156.350 & -0.035 & -2.32 & -0.05 \\
\hline 7 & 4 & 4 & 6 & 5 & 1 & 46753.320 & 0.025 & -16.78 & 0.01 \\
\hline 6 & 2 & 5 & 7 & 1 & 6 & 56040.410 & -0.082 & -13.72 & -0.05 \\
\hline 7 & 0 & 7 & 7 & 1 & 6 & 43362.270 & -0.052 & -9.74 & -0.02 \\
\hline 7 & 1 & 6 & 7 & 2 & 5 & 36433.590 & -0.231 & 0.35 & -0.08 \\
\hline 7 & 2 & 6 & 7 & 2 & 5 & 12094.080 & -0.267 & -7.01 & -0.01 \\
\hline 8 & 4 & 5 & 7 & 5 & 2 & 34783.150 & -0.166 & -9.95 & 0.01 \\
\hline 8 & 0 & 8 & 8 & 1 & 7 & 52868.500 & 0.020 & -16.28 & \\
\hline 8 & 1 & 8 & 8 & 1 & 7 & 50860.990 & 0.107 & -18.46 & 0.02 \\
\hline 7 & 3 & 5 & 8 & 2 & 6 & 34958.940 & -0.058 & -21.63 & -0.04 \\
\hline 8 & 1 & 7 & 8 & 2 & 6 & 38582.200 & -0.264 & -2.67 & -0.12 \\
\hline 7 & 3 & 4 & 8 & 2 & 7 & 15271.100 & -0.121 & -7.45 & -0.03 \\
\hline 8 & 2 & 6 & 8 & 3 & 5 & 61064.090 & 0.156 & 8.60 & -0.27 \\
\hline 9 & 4 & 6 & 8 & 5 & 3 & 22715.350 & -0.138 & -1.23 & 0.01 \\
\hline 9 & 4 & 5 & 8 & 5 & 4 & 22436.410 & -0.141 & -0.66 & 0.01 \\
\hline 9 & 1 & 8 & 9 & 2 & 7 & 42385.570 & -0.063 & -8.69 & -0.16 \\
\hline 9 & 2 & 8 & 9 & 2 & 7 & 26575.450 & 0.071 & -22.13 & 0.01 \\
\hline 10 & 4 & 6 & 9 & 5 & 5 & 9917.780 & -0.094 & 11.18 & 0.02 \\
\hline 10 & 2 & 9 & 10 & 2 & 8 & 35995.460 & 0.243 & -34.42 & 0.04 \\
\hline 9 & 4 & 6 & 10 & 3 & 7 & 24930.210 & -0.061 & -35.51 & -0.03 \\
\hline 9 & 4 & 5 & 10 & 3 & 8 & 16662.440 & -0.052 & -20.61 & -0.04 \\
\hline 11 & 5 & 7 & 10 & 6 & 4 & 27705.670 & -0.063 & -1.73 & 0.01 \\
\hline 11 & 5 & 6 & 10 & 6 & 5 & 27642.480 & -0.236 & -1.50 & 0.01 \\
\hline 11 & 1 & 10 & 11 & 2 & 9 & 55370.420 & -0.288 & -33.31 & -0.17 \\
\hline 10 & 4 & 7 & 11 & 3 & 8 & 40926.870 & 0.007 & -56.71 & -0.01 \\
\hline 11 & 2 & 9 & 11 & 3 & 8 & 54396.250 & -0.194 & 10.60 & -0.78 \\
\hline 11 & 3 & 9 & 11 & 3 & 8 & 12920.070 & -0.072 & -26.43 & 0.06 \\
\hline 10 & 4 & 6 & 11 & 3 & 9 & 27373.550 & -0.120 & -28.67 & -0.08 \\
\hline 12 & 5 & 8 & 11 & 6 & 5 & 15470.060 & 0.212 & 14.46 & 0.01 \\
\hline 12 & 5 & 7 & 11 & 6 & 6 & 15318.530 & -0.123 & 15.14 & 0.01 \\
\hline 12 & 6 & 7 & 11 & 7 & 4 & 44888.660 & 0.019 & -21.70 & 0.02 \\
\hline 11 & 4 & 8 & 12 & 3 & 9 & 58081.220 & 0.217 & -83.57 & 0.01 \\
\hline 12 & 2 & 10 & 12 & 3 & 9 & 54560.050 & -0.072 & 2.89 & -0.98 \\
\hline 11 & 5 & 7 & 12 & 4 & 8 & 16480.930 & -0.057 & -46.87 & -0.01 \\
\hline 11 & 5 & 6 & 12 & 4 & 9 & 13869.530 & -0.080 & -37.44 & -0.04 \\
\hline 13 & 6 & 8 & 12 & 7 & 5 & 32763.160 & -0.191 & -2.61 & \\
\hline 13 & 6 & 7 & 12 & 7 & 6 & 32749.840 & -0.028 & -2.52 & \\
\hline 13 & 2 & 11 & 13 & 3 & 10 & 56501.540 & -0.069 & -11.92 & -1.13 \\
\hline 12 & 5 & 7 & 13 & 4 & 10 & 25984.550 & -0.122 & -54.03 & -0.06 \\
\hline 14 & 6 & 9 & 13 & 7 & 6 & 20528.870 & -0.035 & 19.81 & -0.03 \\
\hline 14 & 6 & 8 & 13 & 7 & 7 & 20495.350 & -0.210 & 20.05 & -0.03 \\
\hline 14 & 2 & 12 & 14 & 3 & 11 & 60423.500 & 0.119 & -35.43 & -1.17 \\
\hline 13 & 5 & 8 & 14 & 4 & 11 & 37858.590 & -0.198 & -71.36 & -0.11 \\
\hline 13 & 6 & 8 & 14 & 5 & 9 & 10051.020 & -0.047 & -60.30 & 0.01 \\
\hline 13 & 6 & 7 & 14 & 5 & 10 & 9358.400 & -0.095 & -56.03 & \\
\hline 15 & 6 & 10 & 14 & 7 & 7 & 8173.100 & -0.149 & 45.90 & -0.05 \\
\hline 15 & 6 & 9 & 14 & 7 & 8 & 8096.410 & -0.073 & 46.54 & -0.05 \\
\hline 15 & 7 & 9 & 14 & 8 & 6 & 37831.930 & -0.171 & -3.87 & -0.05 \\
\hline 15 & 7 & 8 & 14 & 8 & 7 & 37829.370 & 0.051 & -3.84 & -0.05 \\
\hline 14 & 5 & 9 & 15 & 4 & 12 & 49296.900 & -0.224 & -87.90 & -0.23 \\
\hline 14 & 6 & 9 & 15 & 5 & 10 & 23269.790 & 0.006 & -91.08 & 0.05 \\
\hline 14 & 6 & 8 & 15 & 5 & 11 & 21920.340 & -0.143 & -81.59 & \\
\hline 16 & 7 & 10 & 15 & 8 & 7 & 25615.460 & -0.027 & 25.65 & -0.11 \\
\hline 16 & 7 & 9 & 15 & 8 & 8 & 25608.460 & 0.009 & 25.72 & -0.11 \\
\hline 15 & 6 & 10 & 16 & 5 & 11 & 37017.150 & 0.138 & -129.73 & 0.16 \\
\hline
\end{tabular}




\begin{tabular}{|c|c|c|c|c|c|c|c|c|c|}
\hline 15 & 6 & 9 & 16 & 5 & 12 & 34525.530 & -0.125 & -110.05 & -0.01 \\
\hline 17 & 7 & 11 & 16 & 8 & 8 & 13292.170 & 0.179 & 59.40 & -0.17 \\
\hline 16 & 6 & 10 & 17 & 5 & 13 & 47094.800 & -0.210 & -140.65 & -0.04 \\
\hline 16 & 7 & 10 & 17 & 6 & 11 & 17275.500 & -0.013 & -114.15 & 0.14 \\
\hline 16 & 7 & 9 & 17 & 6 & 12 & 16935.070 & -0.113 & -110.46 & 0.12 \\
\hline 18 & 9 & 10 & 17 & 10 & 7 & 60072.230 & -0.137 & -49.42 & -0.17 \\
\hline 18 & 9 & 9 & 17 & 10 & 8 & 60072.230 & -0.097 & -49.42 & -0.17 \\
\hline 17 & 6 & 11 & 18 & 5 & 14 & 59510.130 & 0.099 & -171.96 & -0.14 \\
\hline 17 & 7 & 11 & 18 & 6 & 12 & 30353.010 & 0.079 & -157.43 & 0.25 \\
\hline 17 & 7 & 10 & 18 & 6 & 13 & 29688.790 & -0.099 & -149.40 & 0.17 \\
\hline 19 & 8 & 12 & 18 & 9 & 9 & 18412.610 & 0.037 & 74.26 & -0.38 \\
\hline 19 & 8 & 11 & 18 & 9 & 10 & 18409.200 & 0.117 & 74.32 & -0.38 \\
\hline 19 & 9 & 11 & 18 & 10 & 8 & 47965.490 & 0.059 & -7.78 & -0.34 \\
\hline 19 & 9 & 10 & 18 & 10 & 9 & 47965.490 & 0.169 & -7.78 & -0.34 \\
\hline 19 & 4 & 16 & 19 & 4 & 15 & 48529.050 & -0.133 & -283.09 & 4.90 \\
\hline 18 & 7 & 11 & 19 & 6 & 14 & 42557.340 & -0.082 & -192.78 & 0.23 \\
\hline 18 & 8 & 11 & 19 & 7 & 12 & 11801.160 & 0.061 & -142.56 & 0.33 \\
\hline 18 & 8 & 10 & 19 & 7 & 13 & 11721.350 & -0.199 & -141.32 & 0.32 \\
\hline 20 & 9 & 12 & 19 & 10 & 9 & 35785.590 & -0.119 & 38.71 & -0.52 \\
\hline 20 & 9 & 11 & 19 & 10 & 10 & 35785.590 & 0.168 & 38.72 & -0.52 \\
\hline 19 & 8 & 12 & 20 & 7 & 13 & 24593.850 & 0.071 & -193.14 & 0.46 \\
\hline 19 & 8 & 11 & 20 & 7 & 14 & 24431.870 & -0.040 & -190.33 & 0.43 \\
\hline 20 & 8 & 13 & 21 & 7 & 14 & 37602.910 & 0.136 & -251.22 & 0.67 \\
\hline 20 & 8 & 12 & 21 & 7 & 15 & 37286.800 & 0.061 & -245.19 & 0.58 \\
\hline 22 & 10 & 13 & 21 & 11 & 10 & 40865.430 & -0.063 & 45.80 & -0.94 \\
\hline 22 & 10 & 12 & 21 & 11 & 11 & 40865.430 & -0.006 & 45.80 & -0.94 \\
\hline 21 & 9 & 13 & 22 & 8 & 14 & 19150.350 & -0.051 & -235.08 & 0.86 \\
\hline 21 & 9 & 12 & 22 & 8 & 15 & 19113.160 & -0.118 & -234.19 & 0.85 \\
\hline 23 & 10 & 14 & 22 & 11 & 11 & 28632.130 & 0.051 & 107.94 & -1.25 \\
\hline 23 & 10 & 13 & 22 & 11 & 12 & 28632.130 & 0.192 & 107.94 & -1.25 \\
\hline 23 & 11 & 13 & 22 & 12 & 10 & 58089.150 & -0.118 & -14.10 & -1.12 \\
\hline 23 & 11 & 12 & 22 & 12 & 11 & 58089.150 & -0.114 & -14.10 & -1.12 \\
\hline 22 & 9 & 14 & 23 & 8 & 15 & 31950.990 & 0.141 & -302.14 & 1.12 \\
\hline 22 & 9 & 13 & 23 & 8 & 16 & 31875.610 & -0.103 & -300.19 & 1.09 \\
\hline 24 & 10 & 15 & 23 & 11 & 12 & 16321.630 & -0.089 & 176.25 & -1.60 \\
\hline 24 & 10 & 14 & 23 & 11 & 13 & 16321.630 & 0.250 & 176.27 & -1.60 \\
\hline 24 & 11 & 14 & 23 & 12 & 11 & 45942.900 & 0.245 & 53.11 & -1.57 \\
\hline 24 & 11 & 13 & 23 & 12 & 12 & 45942.900 & 0.256 & 53.11 & -1.57 \\
\hline 23 & 9 & 15 & 24 & 8 & 16 & 44928.280 & 0.316 & -377.50 & 1.49 \\
\hline 23 & 9 & 14 & 24 & 8 & 17 & 44781.340 & 0.101 & -373.35 & 1.40 \\
\hline 23 & 10 & 14 & 24 & 9 & 15 & 13826.990 & -0.156 & -282.03 & 1.48 \\
\hline 23 & 10 & 13 & 24 & 9 & 16 & 13819.020 & 0.016 & -281.78 & 1.48 \\
\hline 25 & 11 & 15 & 24 & 12 & 12 & 33733.490 & 0.001 & 126.57 & -2.04 \\
\hline 25 & 11 & 14 & 24 & 12 & 13 & 33733.490 & 0.029 & 126.57 & -2.04 \\
\hline 24 & 9 & 16 & 25 & 8 & 17 & 58113.650 & 0.015 & -462.57 & 2.04 \\
\hline 24 & 9 & 15 & 25 & 8 & 18 & 57836.340 & -0.148 & -454.11 & 1.81 \\
\hline 24 & 10 & 15 & 25 & 9 & 16 & 26503.080 & 0.112 & -359.87 & 1.84 \\
\hline 24 & 10 & 14 & 25 & 9 & 17 & 26485.930 & -0.061 & -359.29 & 1.83 \\
\hline 26 & 11 & 16 & 25 & 12 & 13 & 21456.550 & 0.001 & 206.69 & -2.55 \\
\hline 26 & 11 & 15 & 25 & 12 & 14 & 21456.550 & 0.068 & 206.69 & -2.55 \\
\hline 26 & 12 & 15 & 25 & 13 & 12 & 51017.890 & 0.075 & 60.52 & -2.48 \\
\hline 26 & 12 & 14 & 25 & 13 & 13 & 51017.890 & 0.077 & 60.52 & -2.48 \\
\hline 25 & 10 & 16 & 26 & 9 & 17 & 39313.070 & 0.186 & -445.90 & 2.29 \\
\hline 25 & 10 & 15 & 26 & 9 & 18 & 39278.700 & 0.030 & -444.63 & 2.26 \\
\hline 25 & 11 & 14 & 26 & 10 & 17 & 8558.140 & 0.191 & -333.31 & 2.39 \\
\hline 27 & 11 & 17 & 26 & 12 & 14 & 9106.250 & -0.014 & 293.92 & -3.09 \\
\hline 27 & 11 & 16 & 26 & 12 & 15 & 9106.250 & 0.144 & 293.93 & -3.09 \\
\hline
\end{tabular}




\begin{tabular}{|c|c|c|c|c|c|c|c|c|c|}
\hline 27 & 12 & 16 & 26 & 13 & 13 & 38831.420 & 0.007 & 146.22 & -3.16 \\
\hline 27 & 12 & 15 & 26 & 13 & 14 & 38831.420 & 0.012 & 146.22 & -3.16 \\
\hline 26 & 10 & 16 & 27 & 9 & 19 & 52207.070 & 0.134 & -538.40 & 2.83 \\
\hline 26 & 11 & 16 & 27 & 10 & 17 & 21146.210 & -0.080 & -423.27 & 2.89 \\
\hline 26 & 11 & 15 & 27 & 10 & 18 & 21142.640 & 0.040 & -423.11 & 2.89 \\
\hline 28 & 12 & 17 & 27 & 13 & 14 & 26584.820 & 0.040 & 239.06 & -3.88 \\
\hline 28 & 12 & 16 & 27 & 13 & 15 & 26584.820 & 0.053 & 239.06 & -3.88 \\
\hline 28 & 13 & 16 & 27 & 14 & 13 & 56091.270 & -0.096 & 67.87 & -3.76 \\
\hline 28 & 13 & 15 & 27 & 14 & 14 & 56091.270 & -0.095 & 67.87 & -3.76 \\
\hline 27 & 11 & 17 & 28 & 10 & 18 & 33842.590 & 0.033 & -521.56 & 3.49 \\
\hline 27 & 11 & 16 & 28 & 10 & 19 & 33834.900 & -0.020 & -521.20 & 3.48 \\
\hline 29 & 12 & 18 & 28 & 13 & 15 & 14273.380 & 0.028 & 339.50 & -4.65 \\
\hline 29 & 12 & 17 & 28 & 13 & 16 & 14273.380 & 0.060 & 339.50 & -4.65 \\
\hline 29 & 13 & 17 & 28 & 14 & 14 & 43926.690 & -0.149 & 166.71 & -4.71 \\
\hline 29 & 13 & 16 & 28 & 14 & 15 & 43926.690 & -0.148 & 166.71 & -4.71 \\
\hline 28 & 11 & 18 & 29 & 10 & 19 & 46659.340 & 0.122 & -629.02 & 4.23 \\
\hline 30 & 13 & 18 & 29 & 14 & 15 & 31708.430 & 0.027 & 273.19 & -5.70 \\
\hline 30 & 13 & 17 & 29 & 14 & 16 & 31708.430 & 0.029 & 273.19 & -5.70 \\
\hline 30 & 14 & 17 & 29 & 15 & 14 & 61163.560 & 0.039 & 74.99 & -5.53 \\
\hline 30 & 14 & 16 & 29 & 15 & 15 & 61163.560 & 0.040 & 74.99 & -5.53 \\
\hline 29 & 11 & 19 & 30 & 10 & 20 & 59609.030 & 0.259 & -746.52 & 5.18 \\
\hline 29 & 11 & 18 & 30 & 10 & 21 & 59579.010 & 0.175 & -744.89 & 5.12 \\
\hline 31 & 13 & 19 & 30 & 14 & 16 & 19432.270 & 0.003 & 387.75 & -6.75 \\
\hline 31 & 13 & 18 & 30 & 14 & 17 & 19432.270 & 0.009 & 387.75 & -6.75 \\
\hline 31 & 14 & 18 & 30 & 15 & 15 & 49020.410 & 0.013 & 187.88 & -6.81 \\
\hline 31 & 14 & 17 & 30 & 15 & 16 & 49020.410 & 0.013 & 187.88 & -6.81 \\
\hline 30 & 12 & 19 & 31 & 11 & 20 & 41157.730 & 0.117 & -724.71 & 6.08 \\
\hline 30 & 12 & 18 & 31 & 11 & 21 & 41154.460 & 0.235 & -724.49 & 6.08 \\
\hline 30 & 13 & 18 & 31 & 12 & 19 & 10563.630 & -0.168 & -565.12 & 6.38 \\
\hline 30 & 13 & 17 & 31 & 12 & 20 & 10563.630 & -0.008 & -565.10 & 6.38 \\
\hline 32 & 14 & 19 & 31 & 15 & 16 & 36828.740 & -0.013 & 308.89 & -8.14 \\
\hline 32 & 14 & 18 & 31 & 15 & 17 & 36828.740 & -0.013 & 308.89 & -8.14 \\
\hline 31 & 13 & 19 & 32 & 12 & 20 & 23100.060 & -0.207 & -691.37 & 7.40 \\
\hline 31 & 13 & 18 & 32 & 12 & 21 & 23100.060 & 0.138 & -691.34 & 7.40 \\
\hline 33 & 14 & 20 & 32 & 15 & 17 & 24585.380 & -0.025 & 438.47 & -9.53 \\
\hline 33 & 14 & 19 & 32 & 15 & 18 & 24585.380 & -0.023 & 438.47 & -9.53 \\
\hline 33 & 15 & 19 & 32 & 16 & 16 & 54112.400 & -0.066 & 209.48 & -9.60 \\
\hline 33 & 15 & 18 & 32 & 16 & 17 & 54112.400 & -0.066 & 209.48 & -9.60 \\
\hline 34 & 14 & 21 & 33 & 15 & 18 & 12286.980 & -0.016 & 577.06 & -11.00 \\
\hline 34 & 14 & 20 & 33 & 15 & 19 & 12286.980 & -0.013 & 577.06 & -11.00 \\
\hline 34 & 15 & 20 & 33 & 16 & 17 & 41946.700 & -0.007 & 345.92 & -11.35 \\
\hline 34 & 15 & 19 & 33 & 16 & 18 & 41946.700 & -0.007 & 345.92 & -11.35 \\
\hline 33 & 14 & 20 & 34 & 13 & 21 & 17783.650 & -0.126 & -784.71 & 10.37 \\
\hline 33 & 14 & 19 & 34 & 13 & 22 & 17783.650 & -0.055 & -784.70 & 10.37 \\
\hline 35 & 15 & 21 & 34 & 16 & 18 & 29734.340 & -0.051 & 491.40 & -13.17 \\
\hline 35 & 15 & 20 & 34 & 16 & 19 & 29734.340 & -0.051 & 491.40 & -13.17 \\
\hline 35 & 16 & 20 & 34 & 17 & 17 & 59203.320 & 0.071 & 231.28 & -13.25 \\
\hline 35 & 16 & 19 & 34 & 17 & 18 & 59203.320 & 0.071 & 231.28 & -13.25 \\
\hline 34 & 13 & 22 & 35 & 12 & 23 & 61255.430 & -0.134 & -1131.40 & 11.59 \\
\hline 34 & 13 & 21 & 35 & 12 & 24 & 61252.660 & 0.060 & -1131.14 & 11.58 \\
\hline 34 & 14 & 21 & 35 & 13 & 22 & 30333.330 & -0.163 & -936.58 & 11.84 \\
\hline 34 & 14 & 20 & 35 & 13 & 23 & 30333.330 & -0.011 & -936.56 & 11.84 \\
\hline 36 & 15 & 22 & 35 & 16 & 19 & 17472.680 & -0.001 & 646.39 & -15.06 \\
\hline 36 & 15 & 21 & 35 & 16 & 20 & 17472.680 & -0.001 & 646.39 & -15.06 \\
\hline 36 & 16 & 21 & 35 & 17 & 18 & 47062.700 & -0.125 & 384.01 & -15.52 \\
\hline 36 & 16 & 20 & 35 & 17 & 19 & 47062.700 & -0.125 & 384.01 & -15.52 \\
\hline 35 & 15 & 21 & 36 & 14 & 22 & 12489.290 & -0.107 & -883.29 & 14.22 \\
\hline
\end{tabular}




\begin{tabular}{|c|c|c|c|c|c|c|c|c|c|}
\hline 35 & 15 & 20 & 36 & 14 & 23 & 12489.290 & -0.093 & -883.29 & 14.22 \\
\hline 37 & 16 & 22 & 36 & 17 & 19 & 34880.330 & 0.004 & 546.28 & -17.84 \\
\hline 37 & 16 & 21 & 36 & 17 & 20 & 34880.330 & 0.004 & 546.28 & -17.84 \\
\hline 36 & 15 & 22 & 37 & 14 & 23 & 24975.020 & -0.092 & -1051.99 & 16.04 \\
\hline 36 & 15 & 21 & 37 & 14 & 24 & 24975.020 & -0.061 & -1051.99 & 16.04 \\
\hline 38 & 17 & 22 & 37 & 18 & 19 & 52177.330 & -0.108 & 422.86 & -20.85 \\
\hline 38 & 17 & 21 & 37 & 18 & 20 & 52177.330 & -0.108 & 422.86 & -20.85 \\
\hline 37 & 15 & 23 & 38 & 14 & 24 & 37531.190 & -0.220 & -1231.85 & 18.06 \\
\hline 37 & 15 & 22 & 38 & 14 & 25 & 37531.190 & -0.155 & -1231.85 & 18.06 \\
\hline 39 & 16 & 24 & 38 & 17 & 21 & 10379.270 & -0.041 & 901.28 & -22.73 \\
\hline 39 & 16 & 23 & 38 & 17 & 22 & 10379.270 & -0.040 & 901.28 & -22.73 \\
\hline 39 & 17 & 23 & 38 & 18 & 20 & 40023.870 & -0.066 & 602.78 & -23.79 \\
\hline 39 & 17 & 22 & 38 & 18 & 21 & 40023.870 & -0.066 & 602.78 & -23.79 \\
\hline 38 & 15 & 24 & 39 & 14 & 25 & 50162.760 & -0.063 & -1423.51 & 20.35 \\
\hline 38 & 15 & 23 & 39 & 14 & 26 & 50162.760 & 0.074 & -1423.50 & 20.35 \\
\hline 38 & 16 & 23 & 39 & 15 & 24 & 19639.420 & -0.045 & -1173.29 & 21.37 \\
\hline 38 & 16 & 22 & 39 & 15 & 25 & 19639.420 & -0.038 & -1173.29 & 21.37 \\
\hline 40 & 17 & 24 & 39 & 18 & 21 & 27830.180 & -0.052 & 793.20 & -26.79 \\
\hline 40 & 17 & 23 & 39 & 18 & 22 & 27830.180 & -0.052 & 793.20 & -26.79 \\
\hline 39 & 16 & 24 & 40 & 15 & 25 & 32130.160 & -0.110 & -1371.35 & 23.81 \\
\hline 39 & 16 & 23 & 40 & 15 & 26 & 32130.160 & -0.097 & -1371.35 & 23.81 \\
\hline 41 & 17 & 25 & 40 & 18 & 22 & 15594.170 & 0.015 & 994.58 & -29.87 \\
\hline 41 & 17 & 24 & 40 & 18 & 23 & 15594.170 & 0.015 & 994.58 & -29.87 \\
\hline 41 & 18 & 24 & 40 & 19 & 21 & 45165.730 & 0.060 & 660.52 & -31.29 \\
\hline 41 & 18 & 23 & 40 & 19 & 22 & 45165.730 & 0.060 & 660.52 & -31.29 \\
\hline 40 & 16 & 25 & 41 & 15 & 26 & 44687.320 & 0.156 & -1581.60 & 26.49 \\
\hline 40 & 16 & 24 & 41 & 15 & 27 & 44687.320 & 0.184 & -1581.59 & 26.49 \\
\hline 40 & 17 & 24 & 41 & 16 & 25 & 14320.720 & 0.117 & -1300.15 & 28.05 \\
\hline 40 & 17 & 23 & 41 & 16 & 26 & 14320.720 & 0.119 & -1300.15 & 28.05 \\
\hline 42 & 18 & 25 & 41 & 19 & 22 & 33004.280 & 0.038 & 869.95 & -34.99 \\
\hline 42 & 18 & 24 & 41 & 19 & 23 & 33004.280 & 0.038 & 869.95 & -34.99 \\
\hline 41 & 16 & 26 & 42 & 15 & 27 & 57313.990 & -0.129 & -1804.67 & 29.50 \\
\hline 41 & 16 & 25 & 42 & 15 & 28 & 57313.990 & -0.071 & -1804.66 & 29.50 \\
\hline 41 & 17 & 25 & 42 & 16 & 26 & 26752.210 & -0.032 & -1517.36 & 30.95 \\
\hline 41 & 17 & 24 & 42 & 16 & 27 & 26752.210 & -0.030 & -1517.36 & 30.95 \\
\hline 42 & 17 & 26 & 43 & 16 & 27 & 39242.390 & -0.048 & -1747.18 & 34.09 \\
\hline 42 & 17 & 25 & 43 & 16 & 28 & 39242.390 & -0.042 & -1747.18 & 34.09 \\
\hline 42 & 18 & 25 & 43 & 17 & 26 & 9014.420 & 0.113 & -1432.20 & 36.32 \\
\hline 42 & 18 & 24 & 43 & 17 & 27 & 9014.420 & 0.113 & -1432.20 & 36.32 \\
\hline 44 & 18 & 27 & 43 & 19 & 24 & 8564.790 & 0.159 & 1323.70 & -42.58 \\
\hline 44 & 18 & 26 & 43 & 19 & 25 & 8564.790 & 0.159 & 1323.70 & -42.58 \\
\hline 43 & 17 & 27 & 44 & 16 & 28 & 51794.340 & -0.150 & -1990.22 & 37.53 \\
\hline 43 & 17 & 26 & 44 & 16 & 29 & 51794.340 & -0.138 & -1990.22 & 37.53 \\
\hline 43 & 18 & 26 & 44 & 17 & 27 & 21391.660 & 0.087 & -1669.50 & 39.76 \\
\hline 43 & 18 & 25 & 44 & 17 & 28 & 21391.660 & 0.087 & -1669.50 & 39.76 \\
\hline 44 & 18 & 27 & 45 & 17 & 28 & 33821.150 & 0.156 & -1919.86 & 43.40 \\
\hline 44 & 18 & 26 & 45 & 17 & 29 & 33821.150 & 0.157 & -1919.86 & 43.40 \\
\hline 46 & 20 & 27 & 45 & 21 & 24 & 43345.720 & 0.159 & 1027.86 & -57.76 \\
\hline 46 & 20 & 26 & 45 & 21 & 25 & 43345.720 & 0.159 & 1027.86 & -57.76 \\
\hline 45 & 18 & 28 & 46 & 17 & 29 & 46305.550 & 0.205 & -2183.88 & 47.32 \\
\hline 45 & 18 & 27 & 46 & 17 & 30 & 46305.550 & 0.208 & -2183.88 & 47.32 \\
\hline 47 & 20 & 28 & 46 & 21 & 25 & 31215.400 & -0.108 & 1290.38 & -63.20 \\
\hline 47 & 20 & 27 & 46 & 21 & 26 & 31215.400 & -0.108 & 1290.38 & -63.20 \\
\hline 47 & 21 & 27 & 46 & 22 & 24 & 60581.400 & 0.236 & 836.38 & -66.68 \\
\hline 47 & 21 & 26 & 46 & 22 & 25 & 60581.400 & 0.236 & 836.38 & -66.68 \\
\hline 46 & 18 & 29 & 47 & 17 & 30 & 58847.540 & -0.017 & -2462.17 & 51.59 \\
\hline 46 & 18 & 28 & 47 & 17 & 31 & 58847.540 & -0.012 & -2462.17 & 51.59 \\
\hline
\end{tabular}




$\begin{array}{rrrrrrrrrrr}48 & 21 & 28 & 47 & 22 & 25 & 48513.000 & -0.280 & 1107.87 & -73.21 \\ 48 & 21 & 27 & 47 & 22 & 26 & 48513.000 & -0.280 & 1107.87 & -73.21 \\ 49 & 20 & 30 & 50 & 19 & 31 & & 35391.460 & -0.136 & -2593.73 & 73.34 \\ 49 & 20 & 29 & 50 & 19 & 32 & 35391.460 & -0.136 & -2593.73 & 73.34 \\ 50 & 20 & 31 & 51 & 19 & 32 & 47801.690 & -0.136 & -2917.93 & 78.51 \\ 50 & 20 & 30 & 51 & 19 & 33 & 47801.690 & -0.136 & -2917.93 & 78.51 \\ 51 & 20 & 32 & 52 & 19 & 33 & 60260.320 & 0.116 & -3257.98 & 84.00 \\ 51 & 20 & 31 & 52 & 19 & 34 & 60260.320 & 0.116 & -3257.98 & 84.00\end{array}$

STOPPED ON DIVERGENCY

$\mathrm{RMS}=0.1278 \quad$ GRAMDET.$=0.21 \mathrm{E}-46$

$\begin{array}{rrrr}\text { ROTATIONAL CONSTANTS } & (\mathrm{MHZ}): \\ 20130.567381 & 6544.668675 & 5064.914010 \\ +- & 0.010591 & 0.003015 & 0.003926\end{array}$

QUARTIC DISTORTION CONSTANTS:

$\begin{array}{rrrrrr} & 5.93404351 & -22.67018247 & 78.97183480 & 2.08129276 & -1.11275618 \\ +- & 0.03694882 & 0.23855673 & 0.06113348 & 0.00867439 & 0.25218532\end{array}$

SEXTIC DISTORTION CONSTANTS:

$\begin{array}{rrrrr}-0.87921950 & -4.90187709 & -20.40473628 & 8.04252704 \\ +- & 0.07602584 & 1.22112568 & 1.11751054 & 0.49269207 \\ & & & & \\ +- & 0.15091486 & -9.73629580 & 32.04007753 & \\ & 0.01117618 & 0.29884071 & 1.24980418 & \end{array}$

SIGNIFICANT DIGITS AND CORRELATION MATRIX:

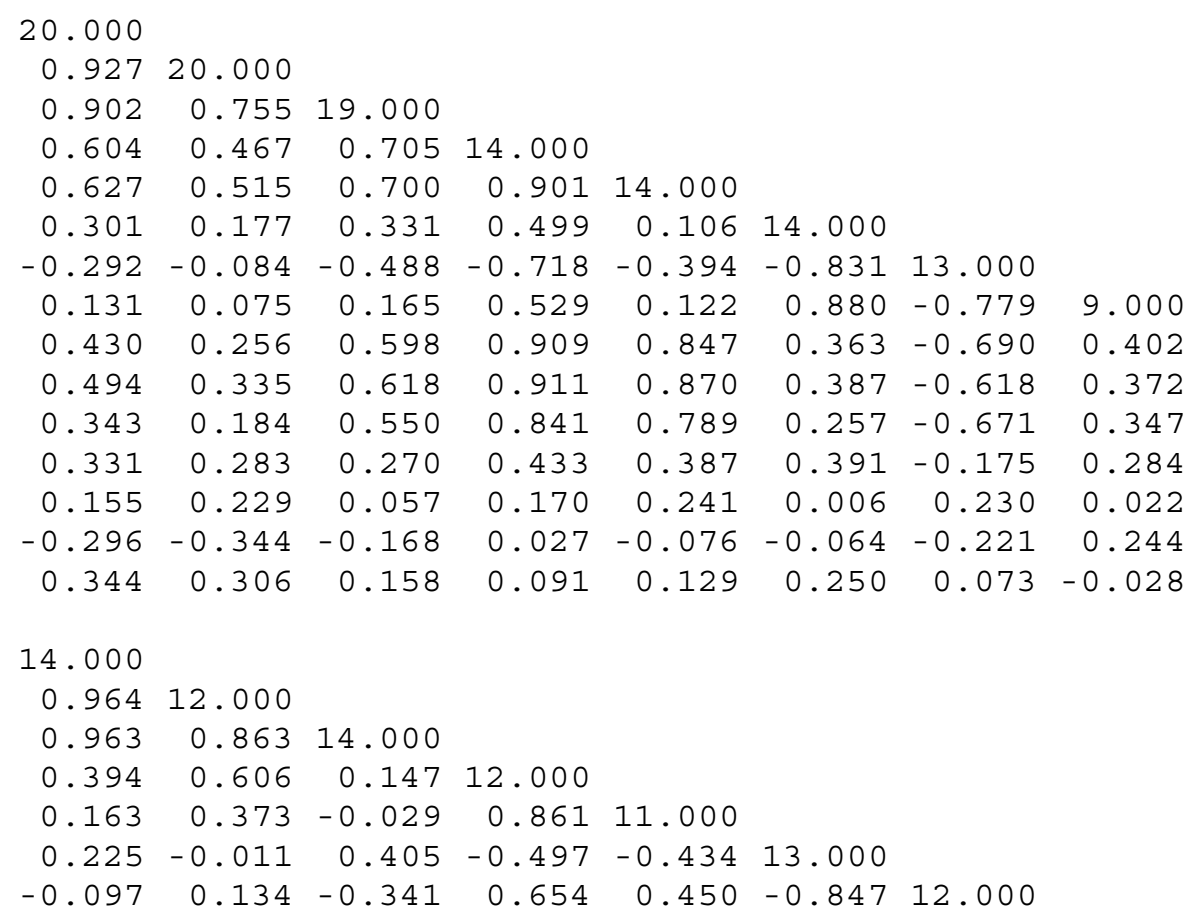


DERIVED CONSTANTS:

$\mathrm{KAPPA}=-0.803559$

MOMENTS OF INERTIA AND DEFECT (U*A**2):

$25.10505742 \quad 77.21995950 \quad 99.78038107 \quad-2.54463585$

+- $\quad 0.000013210 .00003557 \quad 0.00007734 \quad 0.00004793$

STANDARD DISTORTION CONSTANTS (KHZ) :

$\begin{array}{rlr}-248.942783 & -40.386516 & -7.085832 \\ 17.730045 & -23.736174 & 25.478337\end{array}$


Table 4S: Microwave Spectrum and Spectroscopic Constants of the First Excited state of the C2-N3 Torsional Vibration of Formic Hydrazide

TOTAL NUMBER OF ACCEPTED TRANSITIONS: 251

ACCURACY : $\pm 0.15 \mathrm{MHZ}$

$\mathrm{RMS}=0.1238 \quad$ GRAMDET.$=0.19 \mathrm{E}-43$

TRANSITION

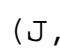

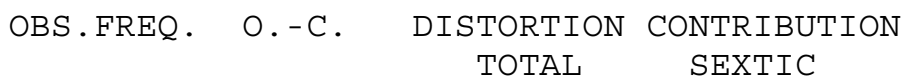

\begin{tabular}{|c|c|c|c|c|c|c|c|c|c|}
\hline$(\mathrm{J}, \mathrm{K}-$ & $1, \mathrm{~K}$ & 7 & & $\mathrm{~K}-1$ & $\mathrm{~K}+1)$ & FREQ. & DELTA & & \\
\hline 1 & 0 & 1 & 1 & 1 & 0 & 14988.000 & 0.093 & -0.05 & \\
\hline 0 & 0 & 0 & 1 & 1 & 1 & 25147.370 & -0.266 & -0.03 & \\
\hline 1 & 0 & 1 & 2 & 0 & 2 & 23202.690 & -0.097 & -0.18 & \\
\hline 1 & 1 & 1 & 2 & 0 & 2 & 9715.820 & -0.008 & -0.17 & \\
\hline 1 & 1 & 0 & 2 & 1 & 1 & 24822.220 & 0.000 & -0.17 & \\
\hline 2 & 0 & 2 & 2 & 1 & 1 & 16607.300 & -0.039 & -0.04 & \\
\hline 1 & 1 & 1 & 2 & 1 & 2 & 21820.230 & -0.191 & 0.01 & \\
\hline 2 & 1 & 2 & 2 & 2 & 1 & 44962.910 & -0.096 & -0.86 & \\
\hline 2 & 0 & 2 & 3 & 0 & 3 & 34512.630 & -0.004 & -0.55 & \\
\hline 2 & 1 & 2 & 3 & 0 & 3 & 22408.130 & 0.089 & -0.73 & \\
\hline 2 & 1 & 1 & 3 & 1 & 2 & 37155.520 & -0.171 & -0.72 & \\
\hline 3 & 0 & 3 & 3 & 1 & 2 & 19250.490 & 0.093 & -0.20 & \\
\hline 2 & 0 & 2 & 3 & 1 & 3 & 44763.690 & 0.004 & -0.04 & \\
\hline 2 & 1 & 2 & 3 & 1 & 3 & 32659.290 & 0.197 & -0.21 & \\
\hline 2 & 2 & 0 & 3 & 2 & 1 & 35451.020 & 0.088 & -0.09 & -0.01 \\
\hline 2 & 2 & 1 & 3 & 2 & 2 & 34982.160 & 0.045 & 0.01 & -0.01 \\
\hline 3 & 0 & 3 & 4 & 0 & 4 & 45499.280 & 0.064 & -1.17 & -0.01 \\
\hline 3 & 1 & 3 & 4 & 0 & 4 & 35248.340 & 0.177 & -1.68 & -0.01 \\
\hline 3 & 1 & 2 & 4 & 1 & 3 & 49387.550 & -0.141 & -1.79 & -0.01 \\
\hline 4 & 0 & 4 & 4 & 1 & 3 & 23139.030 & 0.158 & -0.82 & -0.01 \\
\hline 3 & 1 & 3 & 4 & 1 & 4 & 43420.110 & 0.235 & -0.68 & -0.01 \\
\hline 3 & 2 & 1 & 4 & 2 & 2 & 47691.610 & -0.108 & -1.01 & -0.02 \\
\hline 4 & 1 & 3 & 4 & 2 & 2 & 37177.760 & -0.188 & 0.76 & -0.02 \\
\hline 3 & 2 & 2 & 4 & 2 & 3 & 46550.060 & 0.140 & -0.63 & -0.02 \\
\hline 4 & 1 & 4 & 4 & 2 & 3 & 50416.190 & 0.118 & -0.57 & -0.02 \\
\hline 3 & 3 & 0 & 4 & 3 & 1 & 46898.420 & -0.010 & 0.33 & -0.05 \\
\hline 4 & 1 & 3 & 5 & 1 & 4 & 61470.540 & -0.006 & -3.48 & -0.03 \\
\hline 4 & 2 & 3 & 5 & 1 & 4 & 26021.850 & 0.216 & -4.73 & -0.01 \\
\hline 5 & 0 & 5 & 5 & 1 & 4 & 28482.480 & 0.153 & -2.32 & -0.01 \\
\hline 5 & 1 & 4 & 5 & 2 & 3 & 35917.430 & -0.118 & 1.42 & -0.04 \\
\hline 5 & 1 & 5 & 5 & 2 & 4 & 54365.780 & 0.230 & -0.89 & -0.05 \\
\hline 5 & 2 & 4 & 6 & 1 & 5 & 41328.840 & 0.115 & -8.79 & -0.03 \\
\hline 6 & 0 & 6 & 6 & 1 & 5 & 35386.840 & -0.027 & -5.16 & -0.02 \\
\hline 6 & 1 & 5 & 6 & 2 & 4 & 35510.930 & -0.029 & 1.49 & -0.07 \\
\hline 6 & 1 & 6 & 6 & 2 & 5 & 59133.250 & -0.151 & -1.85 & -0.08 \\
\hline 7 & 4 & 4 & 6 & 5 & 1 & 45569.090 & 0.084 & -17.48 & 0.01 \\
\hline 7 & 4 & 3 & 6 & 5 & 2 & 45529.770 & -0.006 & -17.42 & 0.01 \\
\hline 6 & 2 & 5 & 7 & 1 & 6 & 56844.230 & -0.019 & -14.04 & -0.06 \\
\hline 7 & 1 & 6 & 7 & 2 & 5 & 36311.630 & -0.117 & 0.30 & -0.12 \\
\hline 8 & 0 & 8 & 8 & 1 & 7 & 53417.700 & -0.149 & -15.79 & -0.03 \\
\hline 7 & 3 & 5 & 8 & 2 & 6 & 36157.410 & -0.018 & -22.73 & -0.03 \\
\hline 8 & 1 & 7 & 8 & 2 & 6 & 38596.550 & -0.167 & -2.99 & -0.17 \\
\hline 7 & 3 & 4 & 8 & 2 & 7 & 15901.740 & -0.003 & -7.11 & -0.05 \\
\hline
\end{tabular}




\begin{tabular}{|c|c|c|c|c|c|c|c|c|c|}
\hline 9 & 4 & 6 & 8 & 5 & 3 & 21404.720 & 0.187 & -1.22 & 0.03 \\
\hline 8 & 3 & 6 & 9 & 2 & 7 & 53140.550 & -0.025 & -35.33 & -0.05 \\
\hline 9 & 1 & 8 & 9 & 2 & 7 & 42575.070 & -0.169 & -9.37 & -0.22 \\
\hline 8 & 3 & 5 & 9 & 2 & 8 & 23377.010 & 0.136 & -8.21 & -0.12 \\
\hline 8 & 4 & 5 & 9 & 3 & 6 & 11390.470 & -0.047 & -20.96 & -0.01 \\
\hline 10 & 4 & 7 & 9 & 5 & 4 & 9183.700 & -0.021 & 9.96 & 0.04 \\
\hline 10 & 4 & 6 & 9 & 5 & 5 & 8494.010 & -0.069 & 11.98 & 0.03 \\
\hline 10 & 5 & 6 & 9 & 6 & 3 & 38283.590 & -0.168 & -15.69 & 0.05 \\
\hline 10 & 5 & 5 & 9 & 6 & 4 & 38257.360 & -0.188 & -15.59 & 0.05 \\
\hline 10 & 1 & 9 & 10 & 2 & 8 & 48375.100 & -0.144 & -19.76 & -0.24 \\
\hline 9 & 3 & 6 & 10 & 2 & 9 & 29032.750 & 0.009 & -6.38 & -0.28 \\
\hline 9 & 4 & 6 & 10 & 3 & 7 & 26429.710 & -0.106 & -37.69 & 0.02 \\
\hline 9 & 4 & 5 & 10 & 3 & 8 & 17752.240 & 0.018 & -20.72 & -0.09 \\
\hline 11 & 5 & 6 & 10 & 6 & 5 & 26027.170 & -0.306 & -1.31 & 0.06 \\
\hline 11 & 6 & 5 & 10 & 7 & 4 & 55148.530 & 0.281 & -39.27 & 0.09 \\
\hline 11 & 2 & 10 & 11 & 2 & 9 & 47669.800 & 0.007 & -51.70 & 0.24 \\
\hline 10 & 3 & 7 & 11 & 2 & 10 & 32484.240 & -0.030 & 0.16 & -0.57 \\
\hline 10 & 4 & 7 & 11 & 3 & 8 & 42628.650 & -0.065 & -59.80 & 0.07 \\
\hline 10 & 4 & 6 & 11 & 3 & 9 & 28439.640 & -0.070 & -28.51 & -0.18 \\
\hline 12 & 5 & 8 & 11 & 6 & 5 & 13789.570 & -0.074 & 15.26 & 0.07 \\
\hline 12 & 5 & 7 & 11 & 6 & 6 & 13623.350 & -0.289 & 16.12 & 0.06 \\
\hline 12 & 2 & 11 & 12 & 2 & 10 & 59475.340 & 0.211 & -70.04 & 0.39 \\
\hline 11 & 3 & 8 & 12 & 2 & 11 & 33442.070 & -0.004 & 13.04 & -1.11 \\
\hline 11 & 4 & 8 & 12 & 3 & 9 & 59995.690 & -0.061 & -87.56 & 0.15 \\
\hline 11 & 4 & 7 & 12 & 3 & 10 & 38236.230 & -0.154 & -34.52 & -0.38 \\
\hline 11 & 5 & 7 & 12 & 4 & 8 & 18160.670 & 0.028 & -49.63 & 0.02 \\
\hline 11 & 5 & 6 & 12 & 4 & 9 & 15358.280 & -0.039 & -38.51 & -0.10 \\
\hline 13 & 6 & 8 & 12 & 7 & 5 & 30860.910 & -0.049 & -2.37 & 0.11 \\
\hline 13 & 6 & 7 & 12 & 7 & 6 & 30845.720 & -0.129 & -2.26 & 0.11 \\
\hline 12 & 3 & 9 & 13 & 2 & 12 & 31771.000 & 0.046 & 33.47 & -1.99 \\
\hline 13 & 3 & 11 & 13 & 3 & 10 & 28892.860 & 0.195 & -73.24 & 0.88 \\
\hline 12 & 4 & 8 & 13 & 3 & 11 & 46783.320 & -0.138 & -36.59 & -0.76 \\
\hline 12 & 5 & 8 & 13 & 4 & 9 & 32586.590 & -0.173 & -77.90 & 0.13 \\
\hline 12 & 5 & 7 & 13 & 4 & 10 & 27510.010 & -0.108 & -55.21 & -0.19 \\
\hline 14 & 6 & 9 & 13 & 7 & 6 & 18553.820 & 0.085 & 21.04 & 0.12 \\
\hline 14 & 2 & 12 & 14 & 3 & 11 & 60786.260 & 0.146 & -39.55 & -1.50 \\
\hline 13 & 4 & 9 & 14 & 3 & 12 & 53672.140 & -0.036 & -31.81 & -1.47 \\
\hline 13 & 5 & 8 & 14 & 4 & 11 & 39395.210 & -0.034 & -72.35 & -0.36 \\
\hline 13 & 6 & 8 & 14 & 5 & 9 & 11944.710 & 0.028 & -63.46 & -0.03 \\
\hline 13 & 6 & 7 & 14 & 5 & 10 & 11185.550 & 0.008 & -58.26 & -0.11 \\
\hline 15 & 7 & 9 & 14 & 8 & 6 & 35637.740 & -0.034 & -3.45 & 0.19 \\
\hline 14 & 4 & 10 & 15 & 3 & 13 & 58478.440 & 0.101 & -16.88 & -2.70 \\
\hline 14 & 5 & 9 & 15 & 4 & 12 & 50806.230 & -0.014 & -88.23 & -0.70 \\
\hline 14 & 6 & 9 & 15 & 5 & 10 & 25294.870 & -0.048 & -95.81 & 0.06 \\
\hline 14 & 6 & 8 & 15 & 5 & 11 & 23817.700 & -0.094 & -84.47 & -0.16 \\
\hline 16 & 7 & 10 & 15 & 8 & 7 & 23348.500 & -0.081 & 27.41 & 0.20 \\
\hline 16 & 7 & 9 & 15 & 8 & 8 & 23340.750 & 0.208 & 27.51 & 0.19 \\
\hline 16 & 8 & 9 & 15 & 9 & 6 & 52597.660 & 0.062 & -39.65 & 0.33 \\
\hline 16 & 8 & 8 & 15 & 9 & 7 & 52597.660 & 0.298 & -39.65 & 0.33 \\
\hline 15 & 5 & 10 & 16 & 4 & 13 & 61474.720 & 0.161 & -100.28 & -1.37 \\
\hline 15 & 6 & 10 & 16 & 5 & 11 & 39207.460 & 0.051 & -136.54 & 0.28 \\
\hline 15 & 6 & 9 & 16 & 5 & 12 & 36484.510 & -0.076 & -113.41 & -0.26 \\
\hline 17 & 7 & 10 & 16 & 8 & 9 & 10929.500 & -0.104 & 62.90 & 0.19 \\
\hline 16 & 7 & 10 & 17 & 6 & 11 & 19530.170 & 0.027 & -119.64 & -0.03 \\
\hline 16 & 7 & 9 & 17 & 6 & 12 & 19149.710 & -0.015 & -115.11 & -0.15 \\
\hline 17 & 4 & 13 & 18 & 3 & 16 & 57222.440 & 0.018 & 113.53 & -12.27 \\
\hline 18 & 4 & 15 & 18 & 4 & 14 & 38658.960 & -0.131 & -225.08 & 6.65 \\
\hline
\end{tabular}




\begin{tabular}{|c|c|c|c|c|c|c|c|c|c|}
\hline 17 & 6 & 11 & 18 & 5 & 14 & 61532.710 & 0.176 & -175.14 & -0.91 \\
\hline 17 & 7 & 11 & 18 & 6 & 12 & 32728.180 & -0.060 & -164.94 & 0.13 \\
\hline 17 & 7 & 10 & 18 & 6 & 13 & 31986.790 & -0.012 & -155.22 & -0.17 \\
\hline 19 & 8 & 12 & 18 & 9 & 9 & 15779.280 & -0.122 & 78.59 & 0.30 \\
\hline 19 & 8 & 11 & 18 & 9 & 10 & 15775.600 & 0.264 & 78.67 & 0.30 \\
\hline 19 & 9 & 11 & 18 & 10 & 8 & 45188.110 & -0.057 & -6.63 & 0.52 \\
\hline 19 & 9 & 10 & 18 & 10 & 9 & 45188.110 & 0.074 & -6.63 & 0.52 \\
\hline 18 & 7 & 12 & 19 & 6 & 13 & 46317.830 & 0.031 & -219.40 & 0.47 \\
\hline 18 & 7 & 11 & 19 & 6 & 14 & 44936.150 & 0.142 & -199.66 & -0.24 \\
\hline 18 & 8 & 10 & 19 & 7 & 13 & 14230.170 & -0.112 & -147.80 & -0.17 \\
\hline 20 & 9 & 12 & 19 & 10 & 9 & 32937.130 & -0.164 & 42.06 & 0.49 \\
\hline 20 & 9 & 11 & 19 & 10 & 10 & 32937.130 & 0.177 & 42.07 & 0.49 \\
\hline 19 & 7 & 13 & 20 & 6 & 14 & 60426.150 & -0.217 & -285.94 & 1.19 \\
\hline 19 & 8 & 12 & 20 & 7 & 13 & 27213.970 & 0.011 & -202.08 & \\
\hline 19 & 8 & 11 & 20 & 7 & 14 & 27029.440 & -0.024 & -198.59 & -0.13 \\
\hline 21 & 10 & 12 & 20 & 11 & 9 & 49959.880 & -0.027 & -8.77 & 0.80 \\
\hline 21 & 10 & 11 & 20 & 11 & 10 & 49959.880 & -0.001 & -8.77 & 0.80 \\
\hline 21 & 5 & 17 & 21 & 5 & 16 & 26274.620 & 0.080 & -287.17 & 13.51 \\
\hline 20 & 8 & 13 & 21 & 7 & 14 & 40336.240 & 0.113 & -262.66 & 0.24 \\
\hline 20 & 8 & 12 & 21 & 7 & 15 & 39976.270 & 0.001 & -255.28 & -0.08 \\
\hline 20 & 9 & 12 & 21 & 8 & 13 & 9301.250 & 0.100 & -183.91 & -0.21 \\
\hline 20 & 9 & 11 & 21 & 8 & 14 & 9280.540 & -0.080 & -183.42 & -0.23 \\
\hline 22 & 10 & 13 & 21 & 11 & 10 & 37726.550 & -0.087 & 50.35 & 0.74 \\
\hline 22 & 10 & 12 & 21 & 11 & 11 & 37726.550 & -0.018 & 50.35 & 0.74 \\
\hline 21 & 9 & 13 & 22 & 8 & 14 & 22040.530 & 0.185 & -246.18 & -0.10 \\
\hline 21 & 9 & 12 & 22 & 8 & 15 & 21997.070 & -0.101 & -245.06 & -0.15 \\
\hline 23 & 10 & 14 & 22 & 11 & 11 & 25420.080 & -0.080 & 115.39 & 0.68 \\
\hline 23 & 10 & 13 & 22 & 11 & 12 & 25420.080 & 0.091 & 115.40 & 0.68 \\
\hline 23 & 11 & 13 & 22 & 12 & 10 & 54729.770 & -0.033 & -11.31 & 1.18 \\
\hline 23 & 11 & 12 & 22 & 12 & 11 & 54729.770 & -0.028 & -11.31 & 1.18 \\
\hline 23 & 5 & 19 & 23 & 5 & 18 & 48413.170 & 0.023 & -526.02 & 29.08 \\
\hline 22 & 9 & 14 & 23 & 8 & 15 & 34939.660 & 0.078 & -315.97 & 0.10 \\
\hline 22 & 9 & 13 & 23 & 8 & 16 & 34852.260 & -0.002 & -313.52 & -0.03 \\
\hline 24 & 11 & 14 & 23 & 12 & 11 & 42513.650 & 0.025 & 59.24 & 1.10 \\
\hline 24 & 11 & 13 & 23 & 12 & 12 & 42513.650 & 0.038 & 59.24 & 1.10 \\
\hline 23 & 9 & 15 & 24 & 8 & 16 & 48025.500 & 0.206 & -394.42 & 0.47 \\
\hline 23 & 10 & 14 & 24 & 9 & 15 & 16995.720 & -0.118 & -295.94 & -0.20 \\
\hline 23 & 10 & 13 & 24 & 9 & 16 & 16985.860 & -0.319 & -295.61 & -0.22 \\
\hline 25 & 11 & 15 & 24 & 12 & 12 & 30232.500 & -0.004 & 136.23 & 1.00 \\
\hline 25 & 11 & 14 & 24 & 12 & 13 & 30232.500 & 0.031 & 136.23 & 1.00 \\
\hline 25 & 12 & 14 & 24 & 13 & 11 & 59498.190 & -0.001 & -14.27 & 1.72 \\
\hline 25 & 12 & 13 & 24 & 13 & 12 & 59498.190 & 0.000 & -14.27 & 1.72 \\
\hline 24 & 9 & 16 & 25 & 8 & 17 & 61332.570 & -0.002 & -483.04 & 1.13 \\
\hline 24 & 10 & 15 & 25 & 9 & 16 & 29763.470 & 0.089 & -376.95 & 0.01 \\
\hline 24 & 10 & 14 & 25 & 9 & 17 & 29743.220 & -0.030 & -376.20 & -0.04 \\
\hline 26 & 11 & 16 & 25 & 12 & 13 & 17880.970 & -0.011 & 220.09 & 0.87 \\
\hline 26 & 11 & 15 & 25 & 12 & 14 & 17880.970 & 0.072 & 220.09 & 0.87 \\
\hline 26 & 12 & 15 & 25 & 13 & 12 & 47298.900 & -0.101 & 68.72 & 1.58 \\
\hline 26 & 12 & 14 & 25 & 13 & 13 & 47298.900 & -0.099 & 68.72 & 1.58 \\
\hline 25 & 10 & 16 & 26 & 9 & 17 & 42671.520 & 0.112 & -466.42 & 0.34 \\
\hline 25 & 10 & 15 & 26 & 9 & 18 & 42630.810 & -0.053 & -464.81 & 0.22 \\
\hline 27 & 12 & 16 & 26 & 13 & 13 & 35041.710 & -0.032 & 158.66 & 1.43 \\
\hline 27 & 12 & 15 & 26 & 13 & 14 & 35041.710 & -0.025 & 158.66 & 1.43 \\
\hline 26 & 11 & 16 & 27 & 10 & 17 & 24684.380 & 0.020 & -444.37 & -0.10 \\
\hline 26 & 11 & 15 & 27 & 10 & 18 & 24679.930 & 0.034 & -444.16 & -0.11 \\
\hline 28 & 12 & 17 & 27 & 13 & 14 & 22721.910 & -0.008 & 255.98 & 1.26 \\
\hline 28 & 12 & 16 & 27 & 13 & 15 & 22721.910 & 0.008 & 255.98 & 1.26 \\
\hline
\end{tabular}




\begin{tabular}{|c|c|c|c|c|c|c|c|c|c|}
\hline 28 & 13 & 16 & 27 & 14 & 13 & 52083.400 & 0.105 & 78.76 & 2.23 \\
\hline 28 & 13 & 15 & 27 & 14 & 14 & 52083.400 & 0.106 & 78.76 & 2.23 \\
\hline 27 & 11 & 17 & 28 & 10 & 18 & 37473.000 & 0.147 & -546.67 & 0.25 \\
\hline 27 & 11 & 16 & 28 & 10 & 19 & 37463.670 & 0.051 & -546.20 & 0.22 \\
\hline 29 & 12 & 18 & 28 & 13 & 15 & 10334.790 & 0.038 & 361.12 & 1.05 \\
\hline 29 & 12 & 17 & 28 & 13 & 16 & 10334.790 & 0.077 & 361.12 & 1.05 \\
\hline 29 & 13 & 17 & 28 & 14 & 14 & 39848.980 & -0.051 & 182.66 & 2.02 \\
\hline 29 & 13 & 16 & 28 & 14 & 15 & 39848.980 & -0.049 & 182.66 & 2.02 \\
\hline 28 & 11 & 18 & 29 & 10 & 19 & 50387.350 & 0.043 & -658.41 & 0.78 \\
\hline 28 & 11 & 17 & 29 & 10 & 20 & 50368.690 & -0.082 & -657.42 & 0.69 \\
\hline 30 & 13 & 18 & 29 & 14 & 15 & 27558.760 & -0.009 & 294.44 & 1.78 \\
\hline 30 & 13 & 17 & 29 & 14 & 16 & 27558.760 & -0.006 & 294.44 & 1.78 \\
\hline 29 & 12 & 17 & 30 & 11 & 20 & 32352.270 & 0.029 & -634.07 & 0.14 \\
\hline 31 & 13 & 19 & 30 & 14 & 16 & 15208.510 & -0.034 & 414.56 & 1.50 \\
\hline 31 & 13 & 18 & 30 & 14 & 17 & 15208.510 & -0.026 & 414.56 & 1.50 \\
\hline 30 & 13 & 18 & 31 & 12 & 19 & 14662.450 & -0.068 & -596.91 & -0.45 \\
\hline 30 & 13 & 17 & 31 & 12 & 20 & 14662.450 & 0.134 & -596.89 & -0.45 \\
\hline 32 & 14 & 19 & 31 & 15 & 16 & 32393.090 & 0.002 & 335.48 & 2.48 \\
\hline 32 & 14 & 18 & 31 & 15 & 17 & 32393.090 & 0.002 & 335.48 & 2.48 \\
\hline 32 & 15 & 18 & 31 & 16 & 15 & 61649.930 & -0.096 & 100.50 & 4.19 \\
\hline 32 & 15 & 17 & 31 & 16 & 16 & 61649.930 & -0.096 & 100.50 & 4.19 \\
\hline 31 & 12 & 20 & 32 & 11 & 21 & 58072.840 & -0.012 & -896.91 & 1.52 \\
\hline 31 & 12 & 19 & 32 & 11 & 22 & 58064.440 & -0.049 & -896.33 & 1.46 \\
\hline 31 & 13 & 19 & 32 & 12 & 20 & 27283.680 & -0.238 & -728.64 & \\
\hline 31 & 13 & 18 & 32 & 12 & 21 & 27283.680 & 0.197 & -728.61 & \\
\hline 33 & 14 & 20 & 32 & 15 & 17 & 20077.270 & 0.006 & 471.58 & 2.12 \\
\hline 33 & 14 & 19 & 32 & 15 & 18 & 20077.270 & 0.008 & 471.58 & 2.12 \\
\hline 33 & 15 & 19 & 32 & 16 & 16 & 49460.800 & 0.020 & 235.33 & 3.80 \\
\hline 33 & 15 & 18 & 32 & 16 & 17 & 49460.800 & 0.020 & 235.33 & 3.80 \\
\hline 32 & 14 & 19 & 33 & 13 & 20 & 9688.910 & -0.018 & -681.73 & -0.76 \\
\hline 32 & 14 & 18 & 33 & 13 & 21 & 9688.910 & 0.024 & -681.73 & -0.76 \\
\hline 34 & 15 & 20 & 33 & 16 & 17 & 37225.980 & -0.006 & 379.07 & 3.39 \\
\hline 34 & 15 & 19 & 33 & 16 & 18 & 37225.980 & -0.005 & 379.07 & 3.39 \\
\hline 33 & 13 & 21 & 34 & 12 & 22 & 52803.870 & -0.389 & -1022.92 & 1.46 \\
\hline 33 & 14 & 20 & 34 & 13 & 21 & 22245.320 & -0.092 & -829.84 & -0.24 \\
\hline 33 & 14 & 19 & 34 & 13 & 22 & 22245.320 & -0.002 & -829.83 & -0.25 \\
\hline 35 & 15 & 21 & 34 & 16 & 18 & 24942.820 & 0.007 & 532.17 & 2.95 \\
\hline 35 & 15 & 20 & 34 & 16 & 19 & 24942.820 & 0.008 & 532.17 & 2.95 \\
\hline 34 & 14 & 21 & 35 & 13 & 22 & 34880.330 & -0.111 & -988.40 & 0.44 \\
\hline 34 & 14 & 20 & 35 & 13 & 23 & 34880.330 & 0.084 & -988.38 & 0.43 \\
\hline 36 & 15 & 22 & 35 & 16 & 19 & 12608.260 & -0.026 & 695.08 & 2.45 \\
\hline 36 & 15 & 21 & 35 & 16 & 20 & 12608.260 & -0.025 & 695.08 & 2.45 \\
\hline 36 & 16 & 21 & 35 & 17 & 18 & 42058.220 & -0.054 & 425.21 & 4.58 \\
\hline 36 & 16 & 20 & 35 & 17 & 19 & 42058.220 & -0.054 & 425.21 & 4.58 \\
\hline 35 & 14 & 22 & 36 & 13 & 23 & 47599.270 & -0.248 & -1158.04 & 1.35 \\
\hline 35 & 14 & 21 & 36 & 13 & 24 & 47599.270 & 0.158 & -1158.00 & 1.34 \\
\hline 35 & 15 & 21 & 36 & 14 & 22 & 17228.820 & -0.010 & -937.73 & -0.63 \\
\hline 35 & 15 & 20 & 36 & 14 & 23 & 17228.820 & 0.008 & -937.73 & -0.63 \\
\hline 37 & 16 & 22 & 36 & 17 & 19 & 29806.520 & -0.057 & 596.32 & 4.03 \\
\hline 37 & 16 & 21 & 36 & 17 & 20 & 29806.520 & -0.057 & 596.32 & 4.03 \\
\hline 37 & 17 & 21 & 36 & 18 & 18 & 59071.950 & 0.021 & 294.16 & 6.72 \\
\hline 37 & 17 & 20 & 36 & 18 & 19 & 59071.950 & 0.021 & 294.16 & 6.72 \\
\hline 36 & 15 & 22 & 37 & 14 & 23 & 29796.920 & 0.003 & -1114.16 & 0.13 \\
\hline 36 & 15 & 21 & 37 & 14 & 24 & 29796.920 & 0.044 & -1114.15 & 0.13 \\
\hline 38 & 16 & 23 & 37 & 17 & 20 & 17508.530 & -0.093 & 777.75 & 3.43 \\
\hline 38 & 16 & 22 & 37 & 17 & 21 & 17508.530 & -0.093 & 777.75 & 3.43 \\
\hline 38 & 17 & 22 & 37 & 18 & 19 & 46890.660 & 0.095 & 473.90 & 6.09 \\
\hline
\end{tabular}




\begin{tabular}{|c|c|c|c|c|c|c|c|c|c|}
\hline 38 & 17 & 21 & 37 & 18 & 20 & 46890.660 & 0.095 & 473.90 & 6.09 \\
\hline 37 & 15 & 23 & 38 & 14 & 24 & 42438.600 & -0.002 & -1302.07 & 1.11 \\
\hline 37 & 15 & 22 & 38 & 14 & 25 & 42438.600 & 0.084 & -1302.06 & 1.11 \\
\hline 37 & 16 & 22 & 38 & 15 & 23 & 12227.670 & 0.132 & -1052.30 & -1.19 \\
\hline 37 & 16 & 21 & 38 & 15 & 24 & 12227.670 & 0.136 & -1052.30 & -1.19 \\
\hline 39 & 17 & 23 & 38 & 18 & 20 & 34669.660 & 0.068 & 664.04 & 5.43 \\
\hline 39 & 17 & 22 & 38 & 18 & 21 & 34669.660 & 0.068 & 664.04 & 5.43 \\
\hline 38 & 15 & 24 & 39 & 14 & 25 & 55158.380 & -0.308 & -1502.10 & 2.40 \\
\hline 38 & 15 & 23 & 39 & 14 & 26 & 55158.380 & -0.129 & -1502.08 & 2.40 \\
\hline 38 & 16 & 23 & 39 & 15 & 24 & 24735.720 & 0.080 & -1247.66 & -0.35 \\
\hline 38 & 16 & 22 & 39 & 15 & 25 & 24735.720 & 0.088 & -1247.66 & -0.35 \\
\hline 40 & 17 & 24 & 39 & 18 & 21 & 22407.020 & 0.193 & 865.02 & 4.72 \\
\hline 40 & 17 & 23 & 39 & 18 & 22 & 22407.020 & 0.193 & 865.02 & 4.72 \\
\hline 39 & 16 & 24 & 40 & 15 & 25 & 37308.780 & 0.029 & -1454.94 & 0.70 \\
\hline 39 & 16 & 23 & 40 & 15 & 26 & 37308.780 & 0.047 & -1454.94 & 0.70 \\
\hline 41 & 17 & 25 & 40 & 18 & 22 & 10100.130 & 0.142 & 1077.33 & 3.95 \\
\hline 41 & 17 & 24 & 40 & 18 & 23 & 10100.130 & 0.142 & 1077.33 & 3.95 \\
\hline 40 & 16 & 25 & 41 & 15 & 26 & 49950.730 & -0.091 & -1674.74 & 2.06 \\
\hline 40 & 16 & 24 & 41 & 15 & 27 & 49950.730 & -0.053 & -1674.74 & 2.06 \\
\hline 40 & 17 & 24 & 41 & 16 & 25 & 19690.350 & 0.238 & -1388.90 & -1.07 \\
\hline 40 & 17 & 23 & 41 & 16 & 26 & 19690.350 & 0.240 & -1388.89 & -1.07 \\
\hline 41 & 17 & 25 & 42 & 16 & 26 & 32201.430 & 0.262 & -1616.64 & 0.06 \\
\hline 41 & 17 & 24 & 42 & 16 & 27 & 32201.430 & 0.265 & -1616.64 & 0.06 \\
\hline 42 & 17 & 26 & 43 & 16 & 27 & 44773.520 & 0.203 & -1857.34 & 1.48 \\
\hline 42 & 17 & 25 & 43 & 16 & 28 & 44773.520 & 0.211 & -1857.34 & 1.48 \\
\hline 42 & 18 & 25 & 43 & 17 & 26 & 14655.380 & -0.220 & -1537.88 & -2.10 \\
\hline 42 & 18 & 24 & 43 & 17 & 27 & 14655.380 & -0.220 & -1537.88 & -2.10 \\
\hline 44 & 19 & 26 & 43 & 20 & 23 & 32201.430 & -0.084 & 1053.41 & 8.47 \\
\hline 44 & 19 & 25 & 43 & 20 & 24 & 32201.430 & -0.084 & 1053.41 & 8.47 \\
\hline 43 & 17 & 27 & 44 & 16 & 28 & 57410.130 & 0.067 & -2111.59 & 3.29 \\
\hline 43 & 17 & 26 & 44 & 16 & 29 & 57410.130 & 0.083 & -2111.59 & 3.29 \\
\hline 43 & 18 & 26 & 44 & 17 & 27 & 27109.270 & -0.236 & -1787.18 & -0.91 \\
\hline 43 & 18 & 25 & 44 & 17 & 28 & 27109.270 & -0.236 & -1787.18 & -0.91 \\
\hline 46 & 20 & 27 & 45 & 21 & 24 & 37099.670 & -0.056 & 1154.59 & 11.08 \\
\hline 46 & 20 & 26 & 45 & 21 & 25 & 37099.670 & -0.056 & 1154.59 & 11.08 \\
\hline 47 & 19 & 29 & 48 & 18 & 30 & 46978.420 & -0.047 & -2552.74 & 1.08 \\
\hline 47 & 19 & 28 & 48 & 18 & 31 & 46978.420 & -0.047 & -2552.74 & 1.08 \\
\hline
\end{tabular}

$\mathrm{RMS}=0.1238 \quad$ GRAMDET.$=0.19 \mathrm{E}-43$

$\begin{array}{ccrr}\text { ROTATIONAL CONSTANTS }(\mathrm{MHZ}): & & \\ 20067.810898 & 6580.844300 & 5079.856387 \\ +- & 0.010284 & 0.003171 & 0.003364\end{array}$

QUARTIC DISTORTION CONSTANTS:

$\begin{array}{rrrrrr}6.01814938 & -27.99508218 & 83.33923962 & 1.99172365 & 6.14366335 \\ +- & 0.01897443 & 0.15101517 & 0.04655135 & 0.00598386 & 0.19192374\end{array}$

SEXTIC DISTORTION CONSTANTS:

$\begin{array}{rrrrr}-1.18448818 & -11.69223406 & -20.91174100 & 4.10330700 \\ +- & 0.04967050 & 0.69542119 & 1.03779825 & 0.60810821 \\ +- & 0.11967895 & -7.74059270 & 21.24701989 & \\ & 0.01364615 & 0.57668332 & 2.48920499 & \end{array}$




SIGNIFICANT DIGITS AND CORRELATION MATRIX:
$\begin{array}{rrrrrrrr}20.000 \\ 0.927 & 20.000 & & & & & \\ 0.877 & 0.725 & 20.000 & & & & & \\ 0.405 & 0.397 & 0.402 & 14.000 & & & & \\ 0.444 & 0.496 & 0.403 & 0.820 & 14.000 & & & \\ 0.205 & 0.028 & 0.212 & 0.043 & -0.446 & 14.000 & & \\ 0.078 & 0.316 & -0.165 & 0.000 & 0.488 & -0.783 & 13.000 & \\ -0.103 & -0.126 & -0.159 & 0.448 & -0.107 & 0.649 & -0.573 & 11.000 \\ -0.004 & -0.092 & 0.117 & 0.631 & 0.487 & -0.054 & -0.133 & 0.310 \\ 0.250 & 0.172 & 0.319 & 0.572 & 0.618 & -0.075 & 0.143 & -0.018 \\ -0.207 & -0.234 & -0.043 & 0.459 & 0.293 & -0.166 & -0.187 & 0.317 \\ 0.343 & 0.303 & 0.256 & 0.079 & 0.223 & 0.139 & 0.258 & -0.222 \\ 0.191 & 0.276 & 0.129 & 0.244 & 0.478 & -0.266 & 0.544 & -0.285 \\ -0.269 & -0.305 & -0.233 & 0.362 & 0.098 & 0.021 & -0.272 & 0.538 \\ 0.396 & 0.349 & 0.245 & -0.153 & 0.015 & 0.194 & 0.196 & -0.290\end{array}$

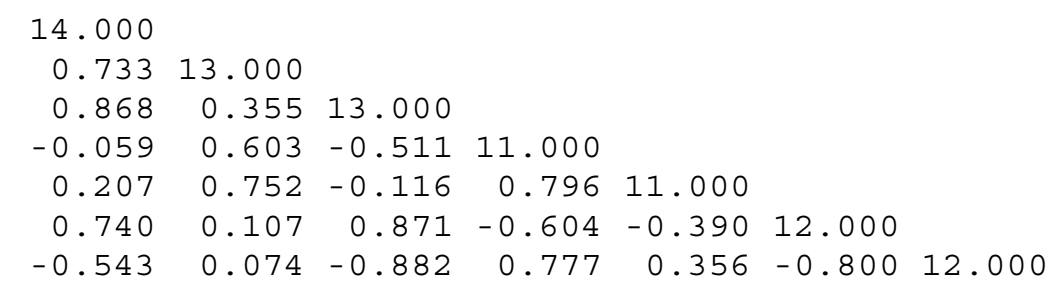

DERIVED CONSTANTS:

$\mathrm{KAPPA}=-0.799707$

MOMENTS OF INERTIA AND DEFECT $(\mathrm{U} * \mathrm{~A} * \star 2)$ :

$\begin{array}{rrrrr}25.18356649 & 76.79547288 & 99.48687748 & -2.49216189 \\ +- & 0.00001291 & 0.00003700 & 0.00006588 & 0.00004168\end{array}$

STANDARD DISTORTION CONSTANTS (KHZ):

$\begin{array}{rrr}-245.449227 & -40.006387 & -8.138808 \\ -0.623981 & -24.072598 & 64.459115\end{array}$


Table 5S: Microwave Spectrum and Spectroscopic Constants of the Second Excited State of the C2-N3 Torsional Vibration of Formic Hydrazide

TOTAL NUMBER OF ACCEPTED TRANSITIONS: 191

ACCURACY : $\pm 0.15 \mathrm{MHZ}$

FIXED CONSTANTS:

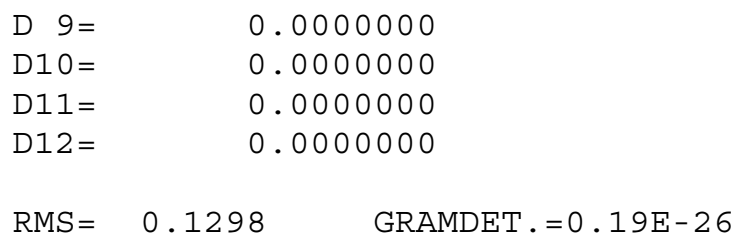

TRANSITION OBS.FREQ. O.-C. DISTORTION CONTRIBUTION TOTAL SEXTIC

\begin{tabular}{|c|c|c|c|c|c|c|c|c|c|}
\hline$(\mathrm{J}, \mathrm{K}-$ & 1,1 & & & $\mathrm{~K}-1$ & $K+1)$ & FREQ. & DELTA & & \\
\hline 2 & 1 & 1 & 2 & 2 & 0 & 40491.850 & -0.232 & -0.62 & \\
\hline 2 & 0 & 2 & 3 & 0 & 3 & 34568.050 & 0.108 & -0.52 & \\
\hline 2 & 1 & 1 & 3 & 1 & 2 & 37232.630 & -0.073 & -0.66 & \\
\hline 3 & 1 & 2 & 3 & 2 & 1 & 38780.020 & -0.163 & 0.04 & -0.01 \\
\hline 3 & 0 & 3 & 4 & 0 & 4 & 45565.540 & 0.053 & -1.07 & -0.01 \\
\hline 3 & 1 & 3 & 4 & 0 & 4 & 35362.240 & 0.229 & -1.60 & \\
\hline 3 & 1 & 2 & 4 & 1 & 3 & 49487.840 & -0.056 & -1.67 & -0.01 \\
\hline 3 & 0 & 3 & 4 & 1 & 4 & 53682.550 & -0.075 & -0.05 & -0.01 \\
\hline 3 & 2 & 1 & 4 & 2 & 2 & 47790.820 & -0.160 & -0.86 & -0.02 \\
\hline 4 & 1 & 3 & 4 & 2 & 2 & 37083.090 & -0.178 & 0.86 & -0.02 \\
\hline 3 & 2 & 2 & 4 & 2 & 3 & 46632.040 & 0.050 & -0.48 & -0.02 \\
\hline 4 & 0 & 4 & 5 & 0 & 5 & 56200.670 & 0.247 & -1.80 & -0.02 \\
\hline 4 & 1 & 3 & 5 & 1 & 4 & 61591.430 & 0.042 & -3.28 & -0.03 \\
\hline 4 & 2 & 3 & 5 & 1 & 4 & 26263.730 & 0.263 & -4.63 & \\
\hline 4 & 1 & 4 & 5 & 1 & 5 & 54161.000 & 0.231 & -1.26 & -0.03 \\
\hline 4 & 2 & 2 & 5 & 2 & 3 & 60341.570 & 0.068 & -2.57 & -0.05 \\
\hline 4 & 2 & 3 & 5 & 2 & 4 & 58139.090 & 0.066 & -1.53 & -0.05 \\
\hline 5 & 1 & 5 & 5 & 2 & 4 & 54376.780 & -0.030 & -0.72 & -0.05 \\
\hline 4 & 3 & 1 & 5 & 3 & 2 & 58885.650 & -0.102 & -0.50 & -0.10 \\
\hline 4 & 3 & 2 & 5 & 3 & 3 & 58761.350 & 0.021 & -0.42 & -0.10 \\
\hline 6 & 4 & 2 & 5 & 5 & 1 & 57141.420 & -0.080 & -22.80 & 0.01 \\
\hline 5 & 1 & 5 & 6 & 0 & 6 & 60442.730 & 0.220 & -4.22 & -0.04 \\
\hline 5 & 2 & 4 & 6 & 1 & 5 & 41607.990 & -0.034 & -8.59 & -0.02 \\
\hline 6 & 0 & 6 & 6 & 1 & 5 & 35542.440 & 0.117 & -5.08 & -0.02 \\
\hline 6 & 1 & 5 & 6 & 2 & 4 & 35452.380 & -0.131 & 1.69 & -0.07 \\
\hline 6 & 1 & 6 & 6 & 2 & 5 & 59178.970 & -0.070 & -1.63 & -0.09 \\
\hline 6 & 2 & 5 & 7 & 1 & 6 & 57156.390 & 0.112 & -13.68 & -0.05 \\
\hline 6 & 3 & 4 & 7 & 2 & 5 & 20331.150 & 0.082 & -12.66 & 0.01 \\
\hline 7 & 1 & 6 & 7 & 2 & 5 & 36295.990 & -0.200 & 0.53 & -0.11 \\
\hline 7 & 3 & 5 & 8 & 2 & 6 & 36608.120 & 0.034 & -22.44 & 0.01 \\
\hline 8 & 1 & 7 & 8 & 2 & 6 & 38641.860 & -0.351 & -2.75 & -0.15 \\
\hline 7 & 3 & 4 & 8 & 2 & 7 & 16088.800 & -0.005 & -6.82 & -0.06 \\
\hline 8 & 2 & 6 & 8 & 3 & 5 & 60201.800 & 0.057 & 10.46 & -0.47 \\
\hline 8 & 3 & 6 & 9 & 2 & 7 & 53652.640 & 0.136 & -34.86 & \\
\hline 8 & 3 & 5 & 9 & 2 & 8 & 23515.870 & -0.045 & -7.69 & -0.16 \\
\hline 8 & 4 & 5 & 9 & 3 & 6 & 11860.500 & -0.040 & -20.77 & 0.05 \\
\hline 9 & 2 & 7 & 9 & 3 & 6 & 57256.130 & 0.267 & 13.59 & -0.69 \\
\hline
\end{tabular}




\begin{tabular}{|c|c|c|c|c|c|c|c|c|c|}
\hline 10 & 5 & 6 & 9 & 6 & 3 & 37781.590 & -0.125 & -15.69 & 0.01 \\
\hline 10 & 5 & 5 & 9 & 6 & 4 & 37754.270 & -0.131 & -15.59 & 0.01 \\
\hline 9 & 4 & 6 & 10 & 3 & 7 & 26978.730 & 0.011 & -37.40 & 0.11 \\
\hline 10 & 2 & 8 & 10 & 3 & 7 & 54982.940 & -0.097 & 14.44 & -0.92 \\
\hline 9 & 4 & 5 & 10 & 3 & 8 & 18113.680 & 0.050 & -20.28 & -0.10 \\
\hline 11 & 5 & 7 & 10 & 6 & 4 & 25565.520 & -0.045 & -1.75 & 0.01 \\
\hline 11 & 1 & 10 & 11 & 2 & 9 & 56319.710 & -0.142 & -34.55 & -0.15 \\
\hline 10 & 4 & 7 & 11 & 3 & 8 & 43268.020 & 0.073 & -59.36 & 0.21 \\
\hline 10 & 4 & 6 & 11 & 3 & 9 & 28787.940 & -0.279 & -27.79 & -0.23 \\
\hline 12 & 5 & 8 & 11 & 6 & 5 & 13227.990 & 0.043 & 14.95 & \\
\hline 12 & 5 & 7 & 11 & 6 & 6 & 13055.120 & 0.099 & 15.83 & -0.02 \\
\hline 12 & 6 & 7 & 11 & 7 & 4 & 42456.880 & -0.002 & -22.39 & 0.03 \\
\hline 11 & 4 & 8 & 12 & 3 & 9 & 60730.640 & 0.266 & -86.90 & 0.34 \\
\hline 11 & 5 & 7 & 12 & 4 & 8 & 18755.390 & -0.132 & -49.22 & 0.15 \\
\hline 11 & 5 & 6 & 12 & 4 & 9 & 15865.180 & -0.097 & -37.92 & -0.08 \\
\hline 13 & 6 & 7 & 12 & 7 & 6 & 30216.850 & -0.031 & -2.48 & \\
\hline 12 & 4 & 8 & 13 & 3 & 11 & 47034.620 & 0.046 & -34.89 & -1.01 \\
\hline 12 & 5 & 8 & 13 & 4 & 9 & 33263.230 & 0.098 & -77.39 & 0.34 \\
\hline 12 & 5 & 7 & 13 & 4 & 10 & 28031.720 & -0.151 & -54.31 & -0.20 \\
\hline 14 & 2 & 12 & 14 & 3 & 11 & 61010.680 & 0.004 & -39.37 & -1.14 \\
\hline 13 & 4 & 9 & 14 & 3 & 12 & 53825.350 & -0.024 & -29.28 & -1.92 \\
\hline 13 & 5 & 8 & 14 & 4 & 11 & 39920.050 & -0.020 & -71.04 & -0.47 \\
\hline 13 & 6 & 8 & 14 & 5 & 9 & 12600.560 & -0.214 & -62.77 & 0.14 \\
\hline 13 & 6 & 7 & 14 & 5 & 10 & 11810.460 & -0.124 & -57.46 & -0.02 \\
\hline 15 & 7 & 9 & 14 & 8 & 6 & 34912.980 & -0.122 & -3.81 & 0.01 \\
\hline 15 & 7 & 8 & 14 & 8 & 7 & 34909.770 & 0.040 & -3.77 & \\
\hline 14 & 5 & 9 & 15 & 4 & 12 & 51316.750 & 0.092 & -86.37 & -0.98 \\
\hline 14 & 6 & 9 & 15 & 5 & 10 & 26009.750 & -0.016 & -94.94 & 0.31 \\
\hline 14 & 6 & 8 & 15 & 5 & 11 & 24473.080 & -0.024 & -83.35 & -0.09 \\
\hline 16 & 7 & 10 & 15 & 8 & 7 & 22592.260 & 0.006 & 26.74 & -0.04 \\
\hline 16 & 7 & 9 & 15 & 8 & 8 & 22583.900 & 0.170 & 26.84 & -0.04 \\
\hline 15 & 6 & 10 & 16 & 5 & 11 & 39996.510 & -0.129 & -135.54 & 0.64 \\
\hline 15 & 6 & 9 & 16 & 5 & 12 & 37166.110 & -0.087 & -111.89 & -0.26 \\
\hline 17 & 7 & 11 & 16 & 8 & 8 & 10158.980 & 0.176 & 61.59 & -0.09 \\
\hline 17 & 7 & 10 & 16 & 8 & 9 & 10138.330 & -0.279 & 61.86 & -0.10 \\
\hline 16 & 6 & 11 & 17 & 5 & 12 & 54758.140 & 0.172 & -187.40 & 1.29 \\
\hline 16 & 6 & 10 & 17 & 5 & 13 & 49799.330 & -0.197 & -142.15 & -0.63 \\
\hline 16 & 7 & 10 & 17 & 6 & 11 & 20313.380 & -0.031 & -118.30 & 0.26 \\
\hline 16 & 7 & 9 & 17 & 6 & 12 & 19913.990 & -0.026 & -113.66 & 0.05 \\
\hline 18 & 8 & 11 & 17 & 9 & 8 & 27291.920 & -0.078 & 33.45 & -0.07 \\
\hline 18 & 8 & 10 & 17 & 9 & 9 & 27290.280 & 0.076 & 33.48 & -0.07 \\
\hline 17 & 7 & 11 & 18 & 6 & 12 & 33565.620 & -0.064 & -163.31 & 0.50 \\
\hline 17 & 7 & 10 & 18 & 6 & 13 & 32787.650 & -0.007 & -153.34 & -0.02 \\
\hline 18 & 7 & 12 & 19 & 6 & 13 & 47220.930 & -0.267 & -217.58 & 0.97 \\
\hline 18 & 8 & 11 & 19 & 7 & 12 & 15189.200 & -0.148 & -147.51 & 0.28 \\
\hline 20 & 9 & 12 & 19 & 10 & 9 & 31987.930 & -0.217 & 40.72 & -0.10 \\
\hline 20 & 9 & 11 & 19 & 10 & 10 & 31987.930 & 0.152 & 40.73 & -0.10 \\
\hline 20 & 10 & 11 & 19 & 11 & 8 & 61142.270 & -0.130 & -63.01 & 0.18 \\
\hline 20 & 10 & 10 & 19 & 11 & 9 & 61142.270 & -0.120 & -63.01 & 0.18 \\
\hline 19 & 7 & 13 & 20 & 6 & 14 & 61411.970 & -0.190 & -284.06 & 1.88 \\
\hline 19 & 7 & 12 & 20 & 6 & 15 & 58823.810 & 0.075 & -244.87 & -0.56 \\
\hline 19 & 8 & 12 & 20 & 7 & 13 & 28127.160 & -0.066 & -199.78 & 0.45 \\
\hline 19 & 8 & 11 & 20 & 7 & 14 & 27931.770 & -0.064 & -196.19 & 0.21 \\
\hline 20 & 8 & 13 & 21 & 7 & 14 & 41300.420 & -0.059 & -259.95 & 0.76 \\
\hline 20 & 8 & 12 & 21 & 7 & 15 & 40919.610 & 0.067 & -252.36 & 0.20 \\
\hline 20 & 9 & 12 & 21 & 8 & 13 & 10261.070 & -0.037 & -181.43 & 0.36 \\
\hline 20 & 9 & 11 & 21 & 8 & 14 & 10238.950 & -0.206 & -180.93 & 0.32 \\
\hline
\end{tabular}




\begin{tabular}{|c|c|c|c|c|c|c|c|c|c|}
\hline 22 & 10 & 13 & 21 & 11 & 10 & 36680.770 & -0.110 & 48.50 & -0.15 \\
\hline 22 & 10 & 12 & 21 & 11 & 11 & 36680.770 & -0.035 & 48.50 & -0.15 \\
\hline 21 & 8 & 13 & 22 & 7 & 16 & 54053.320 & 0.328 & -314.52 & 0.11 \\
\hline 21 & 9 & 13 & 22 & 8 & 14 & 23041.380 & 0.078 & -243.13 & 0.52 \\
\hline 21 & 9 & 12 & 22 & 8 & 15 & 22995.100 & -0.052 & -241.98 & 0.42 \\
\hline 23 & 10 & 14 & 22 & 11 & 11 & 24341.360 & -0.072 & 112.84 & -0.33 \\
\hline 23 & 10 & 13 & 22 & 11 & 12 & 24341.360 & 0.115 & 112.85 & -0.33 \\
\hline 22 & 9 & 14 & 23 & 8 & 15 & 35985.240 & 0.041 & -312.35 & 0.75 \\
\hline 22 & 9 & 13 & 23 & 8 & 16 & 35891.880 & -0.013 & -309.83 & 0.52 \\
\hline 24 & 10 & 15 & 23 & 11 & 12 & 11920.350 & -0.192 & 183.49 & -0.52 \\
\hline 24 & 10 & 14 & 23 & 11 & 13 & 11920.350 & 0.255 & 183.51 & -0.53 \\
\hline 24 & 11 & 14 & 23 & 12 & 11 & 41371.000 & -0.065 & 56.74 & -0.22 \\
\hline 24 & 11 & 13 & 23 & 12 & 12 & 41371.000 & -0.050 & 56.74 & -0.22 \\
\hline 23 & 10 & 14 & 24 & 9 & 15 & 18089.080 & 0.004 & -292.01 & 0.69 \\
\hline 23 & 10 & 13 & 24 & 9 & 16 & 18078.490 & -0.163 & -291.67 & 0.65 \\
\hline 25 & 11 & 15 & 24 & 12 & 12 & 29056.970 & -0.088 & 132.88 & -0.47 \\
\hline 25 & 11 & 14 & 24 & 12 & 13 & 29056.970 & -0.050 & 132.88 & -0.47 \\
\hline 25 & 12 & 14 & 24 & 13 & 11 & 58290.640 & 0.327 & -16.67 & 0.04 \\
\hline 25 & 12 & 13 & 24 & 13 & 12 & 58290.640 & 0.328 & -16.67 & 0.04 \\
\hline 24 & 10 & 15 & 25 & 9 & 16 & 30898.430 & 0.101 & -372.29 & 0.91 \\
\hline 24 & 10 & 14 & 25 & 9 & 17 & 30876.520 & -0.092 & -371.53 & 0.82 \\
\hline 26 & 12 & 15 & 25 & 13 & 12 & 46059.450 & 0.031 & 65.38 & -0.30 \\
\hline 26 & 12 & 14 & 25 & 13 & 13 & 46059.450 & 0.034 & 65.38 & -0.30 \\
\hline 27 & 12 & 16 & 26 & 13 & 13 & 33769.230 & -0.070 & 154.31 & -0.65 \\
\hline 27 & 12 & 15 & 26 & 13 & 14 & 33769.230 & -0.062 & 154.31 & -0.65 \\
\hline 26 & 10 & 17 & 27 & 9 & 18 & 56966.570 & 0.105 & -559.33 & 1.73 \\
\hline 26 & 10 & 16 & 27 & 9 & 19 & 56881.280 & 0.102 & -555.91 & 1.28 \\
\hline 26 & 11 & 16 & 27 & 10 & 17 & 25911.910 & -0.045 & -438.51 & 1.20 \\
\hline 26 & 11 & 15 & 27 & 10 & 18 & 25907.070 & -0.023 & -438.29 & 1.17 \\
\hline 28 & 12 & 17 & 27 & 13 & 14 & 21415.360 & -0.015 & 250.54 & -1.01 \\
\hline 28 & 12 & 16 & 27 & 13 & 15 & 21415.360 & 0.003 & 250.54 & -1.01 \\
\hline 28 & 13 & 16 & 27 & 14 & 13 & 50746.370 & -0.069 & 74.38 & -0.40 \\
\hline 28 & 13 & 15 & 27 & 14 & 14 & 50746.370 & -0.069 & 74.38 & -0.40 \\
\hline 27 & 11 & 17 & 28 & 10 & 18 & 38742.920 & 0.022 & -539.93 & 1.53 \\
\hline 27 & 11 & 16 & 28 & 10 & 19 & 38732.920 & 0.080 & -539.45 & 1.45 \\
\hline 29 & 13 & 17 & 28 & 14 & 14 & 38479.220 & -0.070 & 177.07 & -0.86 \\
\hline 29 & 13 & 16 & 28 & 14 & 15 & 38479.220 & -0.068 & 177.07 & -0.86 \\
\hline 28 & 11 & 18 & 29 & 10 & 19 & 51702.600 & 0.128 & -650.81 & 1.97 \\
\hline 28 & 11 & 17 & 29 & 10 & 20 & 51682.360 & 0.071 & -649.79 & 1.81 \\
\hline 30 & 13 & 18 & 29 & 14 & 15 & 26154.980 & -0.022 & 287.57 & -1.35 \\
\hline 30 & 13 & 17 & 29 & 14 & 16 & 26154.980 & -0.018 & 287.57 & -1.35 \\
\hline 29 & 12 & 18 & 30 & 11 & 19 & 33717.200 & -0.006 & -625.84 & 1.99 \\
\hline 31 & 13 & 19 & 30 & 14 & 16 & 13769.470 & -0.064 & 406.33 & -1.84 \\
\hline 31 & 13 & 18 & 30 & 14 & 17 & 13769.470 & -0.055 & 406.33 & -1.84 \\
\hline 31 & 14 & 18 & 30 & 15 & 15 & 43187.770 & -0.037 & 201.11 & -1.13 \\
\hline 31 & 14 & 17 & 30 & 15 & 16 & 43187.770 & -0.037 & 201.11 & -1.13 \\
\hline 30 & 12 & 19 & 31 & 11 & 20 & 46562.240 & -0.207 & -750.92 & 2.45 \\
\hline 30 & 13 & 18 & 31 & 12 & 19 & 16079.720 & -0.058 & -587.87 & 2.17 \\
\hline 30 & 13 & 17 & 31 & 12 & 20 & 16079.720 & 0.166 & -587.85 & 2.17 \\
\hline 32 & 14 & 19 & 31 & 15 & 16 & 30891.660 & -0.036 & 326.86 & -1.76 \\
\hline 32 & 14 & 18 & 31 & 15 & 17 & 30891.660 & -0.035 & 326.86 & -1.76 \\
\hline 32 & 15 & 18 & 31 & 16 & 15 & 60117.770 & 0.056 & 93.20 & -0.65 \\
\hline 32 & 15 & 17 & 31 & 16 & 16 & 60117.770 & 0.056 & 93.20 & -0.65 \\
\hline 31 & 12 & 20 & 32 & 11 & 21 & 59524.540 & 0.045 & -886.51 & 3.06 \\
\hline 31 & 12 & 19 & 32 & 11 & 22 & 59515.360 & 0.066 & -885.91 & 2.94 \\
\hline 31 & 13 & 19 & 32 & 12 & 20 & 28740.980 & -0.274 & -718.34 & 2.62 \\
\hline 31 & 13 & 18 & 32 & 12 & 21 & 28740.980 & 0.210 & -718.31 & 2.61 \\
\hline
\end{tabular}




\begin{tabular}{|c|c|c|c|c|c|c|c|c|}
\hline 3314 & 20 & 32 & 15 & 17 & 18540.730 & -0.004 & 461.36 & -2.40 \\
\hline 3314 & 19 & 32 & 15 & 18 & 18540.730 & -0.002 & 461.36 & -2.40 \\
\hline $32 \quad 14$ & 19 & 33 & 13 & 20 & 11202.310 & -0.080 & -670.61 & 2.86 \\
\hline 214 & 18 & 33 & 13 & 21 & 11202.310 & -0.034 & -670.60 & 2.86 \\
\hline $4 \quad 15$ & 20 & 33 & 16 & 17 & 35626.510 & -0.021 & 368.33 & -2.26 \\
\hline 415 & 19 & 33 & 16 & 18 & 35626.510 & -0.021 & 368.33 & -2.26 \\
\hline 313 & 21 & 34 & 12 & 22 & 54347.300 & -0.002 & -1010.15 & 3.78 \\
\hline 313 & 20 & 34 & 12 & 23 & 54345.470 & 0.247 & -1009.99 & 3.74 \\
\hline 14 & 20 & 34 & 13 & 21 & 23798.240 & -0.020 & -817.22 & 3.42 \\
\hline 314 & 19 & 34 & 13 & 22 & 23798.240 & 0.082 & -817.21 & 3.41 \\
\hline $5 \quad 15$ & 21 & 34 & 16 & 18 & 23308.280 & 0.029 & 519.55 & -3.08 \\
\hline $5 \quad 15$ & 20 & 34 & 16 & 19 & 23308.280 & 0.029 & 519.55 & -3.08 \\
\hline $4 \quad 14$ & 21 & 35 & 13 & 22 & 36474.090 & -0.152 & -974.29 & 4.03 \\
\hline $4 \quad 14$ & 20 & 35 & 13 & 23 & 36474.090 & 0.067 & -974.27 & 4.03 \\
\hline 515 & 21 & 36 & 14 & 22 & 18878.100 & -0.034 & -922.35 & 4.41 \\
\hline 515 & 20 & 36 & 14 & 23 & 18878.100 & -0.012 & -922.35 & 4.4 \\
\hline 716 & 22 & 36 & 17 & 19 & 28073.460 & 0.030 & 580.84 & -3.89 \\
\hline 3716 & 21 & 36 & 17 & 20 & 28073.460 & 0.030 & 580.84 & -3.89 \\
\hline $37 \quad 17$ & 21 & 36 & 18 & 18 & 57309.180 & 0.011 & 280.24 & -2.26 \\
\hline $37 \quad 17$ & 20 & 36 & 18 & 19 & 57309.180 & 0.011 & 280.24 & -2.26 \\
\hline 3615 & 22 & 37 & 14 & 23 & 31486.420 & -0.079 & -1097.00 & 5.15 \\
\hline 3615 & 21 & 37 & 14 & 24 & 31486.420 & -0.033 & -1097.00 & 5.15 \\
\hline 3816 & 23 & 37 & 17 & 20 & 15739.040 & -0.273 & 759.98 & -4.94 \\
\hline 3816 & 22 & 37 & 17 & 21 & 15739.040 & -0.273 & 759.98 & -4.94 \\
\hline $38 \quad 17$ & 22 & 37 & 18 & 19 & 45093.520 & 0.043 & 457.57 & -3.54 \\
\hline $38 \quad 17$ & 21 & 37 & 18 & 20 & 45093.520 & 0.043 & 457.57 & -3.54 \\
\hline 3715 & 23 & 38 & 14 & 24 & 44169.690 & -0.247 & -1283.16 & 5.98 \\
\hline 3715 & 22 & 38 & 14 & 25 & 44169.690 & -0.149 & -1283.15 & 5.98 \\
\hline $39 \quad 17$ & 23 & 38 & 18 & 20 & 32837.430 & 0.179 & 645.15 & -4.84 \\
\hline 3917 & 22 & 38 & 18 & 21 & 32837.430 & 0.179 & 645.15 & -4.84 \\
\hline $40 \quad 18$ & 23 & 39 & 19 & 20 & 49826.470 & -0.075 & 505.21 & -4.35 \\
\hline $40 \quad 18$ & 22 & 39 & 19 & 21 & 49826.470 & -0.075 & 505.21 & -4.35 \\
\hline 3916 & 24 & 40 & 15 & 25 & 39136.220 & -0.068 & -1432.05 & 7.50 \\
\hline 3916 & 23 & 40 & 15 & 26 & 39136.220 & -0.048 & -1432.05 & 7.50 \\
\hline $41 \quad 18$ & 24 & 40 & 19 & 21 & 37600.470 & 0.028 & 712.42 & -5.95 \\
\hline $41 \quad 18$ & 23 & 40 & 19 & 22 & 37600.470 & 0.028 & 712.42 & -5.95 \\
\hline $40 \quad 16$ & 25 & 41 & 15 & 26 & 51820.790 & -0.049 & -1649.79 & 8.60 \\
\hline 4016 & 24 & 41 & 15 & 27 & 51820.790 & -0.005 & -1649.79 & 8.59 \\
\hline $41 \quad 17$ & 25 & 42 & 16 & 26 & 34126.260 & 0.137 & -1589.02 & 9.3 \\
\hline $41 \quad 17$ & 24 & 42 & 16 & 27 & 34126.260 & 0.141 & -1589.02 & 9.3 \\
\hline
\end{tabular}

STOPPED ON DIVERGENCY

$\mathrm{RMS}=0.1298 \quad$ GRAMDET.$=0.19 \mathrm{E}-26$

ROTATIONAL CONSTANTS (MHZ) :

$\begin{array}{rrrr}20053.957270 & 6596.487778 & 5085.056071 \\ +- & 0.012129 & 0.003516 & 0.004788\end{array}$

QUARTIC DISTORTION CONSTANTS:

$\begin{array}{rrrrrr} & 5.63650672 & -29.64541107 & 82.45257796 & 2.00750491 & 5.00038360 \\ + & 0.03483998 & 0.25580888 & 0.06585619 & 0.00650879 & 0.28483905\end{array}$

SEXTIC DISTORTION CONSTANTS: 


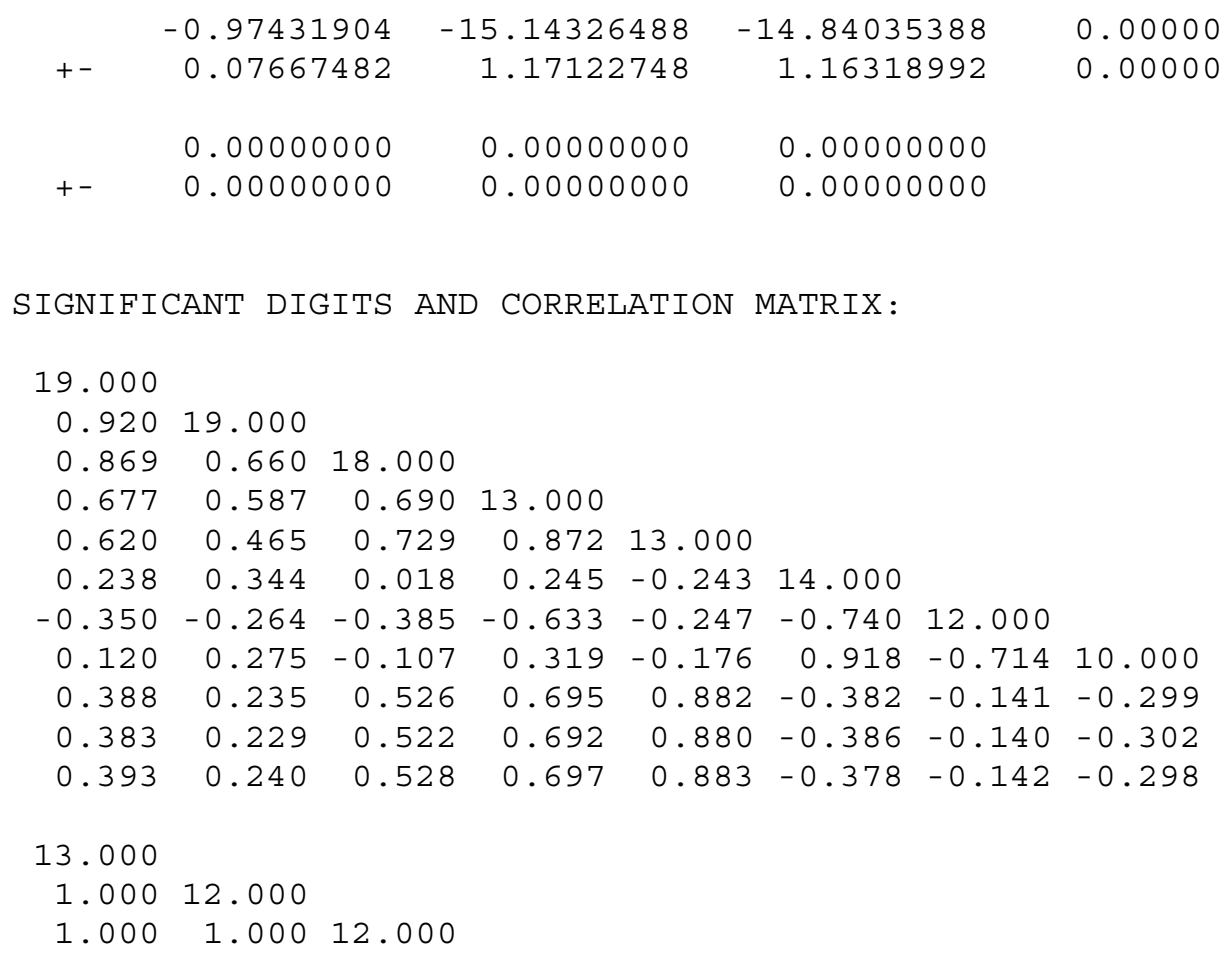

DERIVED CONSTANTS :

$\mathrm{KAPPA}=-0.798057$

MOMENTS OF INERTIA AND DEFECT (U*A**2): $\begin{array}{rrrrr}25.20096374 & 76.61335350 & 99.38514796 & -2.42916928 \\ +-\quad 0.00001524 & 0.00004083 & 0.00009359 & 0.00006542\end{array}$

STANDARD DISTORTION CONSTANTS (KHZ):

$\begin{array}{rrr}-233.774694 & -38.606066 & -6.485988 \\ 8.713241 & -22.546027 & 64.776349\end{array}$


Table 6S: Microwave Spectrum and Spectroscopic Constants of the Third Excited State of the C2-N3 Torsional Vibration of Formic Hydrazide

TOTAL NUMBER OF ACCEPTED TRANSITIONS: 92

ACCURACY : $\pm 0.15 \mathrm{MHZ}$

FIXED CONSTANTS:

$\begin{array}{ll}\mathrm{D} 7= & 0.0000000 \\ \mathrm{D} 8= & 0.0000000 \\ \mathrm{D} 9= & 0.0000000 \\ \mathrm{D} 10= & 0.0000000 \\ \mathrm{D} 11= & 0.0000000 \\ \mathrm{D} 12= & 0.0000000\end{array}$

$\mathrm{RMS}=0.1660 \quad$ GRAMDET.$=0.96 \mathrm{E}-14$

TRANSITION OBS.FREQ. O.-C. DISTORTION CONTRIBUTION TOTAL SEXTIC

\begin{tabular}{|c|c|c|c|c|c|c|c|c|c|}
\hline$(\mathrm{J}, \mathrm{K}-$ & $1, \mathrm{~K}$ & $i+1$ & $>J$ & $\mathrm{~K}-1$ & $K+1)$ & FREQ. & DELTA & & \\
\hline 2 & 0 & 2 & 3 & 0 & 3 & 34558.140 & 0.117 & -0.55 & \\
\hline 2 & 1 & 1 & 3 & 1 & 2 & 37223.810 & 0.122 & -0.69 & \\
\hline 2 & 1 & 2 & 3 & 1 & 3 & 32693.790 & 0.146 & -0.23 & \\
\hline 3 & 1 & 2 & 3 & 2 & 1 & 38842.760 & -0.111 & -0.07 & \\
\hline 3 & 0 & 3 & 4 & 0 & 4 & 45552.520 & 0.083 & -1.16 & \\
\hline 3 & 1 & 3 & 4 & 0 & 4 & 35329.500 & -0.199 & -1.65 & \\
\hline 4 & 0 & 4 & 4 & 1 & 3 & 23212.940 & 0.324 & -0.73 & \\
\hline 3 & 1 & 3 & 4 & 1 & 4 & 43464.030 & -0.110 & -0.71 & \\
\hline 4 & 1 & 3 & 4 & 2 & 2 & 37143.470 & -0.284 & 0.69 & \\
\hline 4 & 1 & 4 & 5 & 0 & 5 & 48049.570 & -0.177 & -2.93 & \\
\hline 4 & 1 & 3 & 5 & 1 & 4 & 61576.760 & 0.100 & $-3 \cdot 34$ & \\
\hline 4 & 1 & 4 & 5 & 1 & 5 & 54142.140 & 0.145 & -1.49 & \\
\hline 4 & 2 & 2 & 5 & 2 & 3 & 60323.920 & 0.139 & -2.68 & \\
\hline 5 & 1 & 4 & 5 & 2 & 3 & 35890.700 & -0.175 & 1.34 & \\
\hline 5 & 1 & 5 & 6 & 0 & 6 & 60408.180 & 0.135 & -4.47 & \\
\hline 6 & 1 & 5 & 6 & 2 & 4 & 35506.390 & 0.021 & 1.47 & \\
\hline 6 & 2 & 5 & 7 & 1 & 6 & 57080.220 & 0.015 & -13.38 & \\
\hline 7 & 0 & 7 & 7 & 1 & 6 & 44035.580 & -0.206 & -8.72 & \\
\hline 6 & 3 & 4 & 7 & 2 & 5 & 20197.250 & 0.050 & -12.07 & \\
\hline 7 & 1 & 6 & 7 & 2 & 5 & 36346.220 & 0.158 & 0.43 & \\
\hline 8 & 1 & 7 & 8 & 2 & 6 & 38688.390 & 0.319 & -2.54 & \\
\hline 8 & 3 & 6 & 9 & 2 & 7 & 53515.780 & -0.116 & -33.50 & -0.01 \\
\hline 9 & 2 & 7 & 9 & 3 & 6 & 57356.140 & 0.009 & 12.50 & \\
\hline 10 & 1 & 9 & 10 & 2 & 8 & 48634.530 & 0.325 & -17.89 & \\
\hline 10 & 2 & 8 & 10 & 3 & 7 & 55076.320 & -0.073 & 13.48 & \\
\hline 9 & 4 & 5 & 10 & 3 & 8 & 17930.940 & -0.008 & -19.85 & -0.02 \\
\hline 10 & 4 & 7 & 11 & 3 & 8 & 43070.520 & 0.022 & -56.87 & -0.03 \\
\hline 11 & 2 & 9 & 11 & 3 & 8 & 53957.960 & -0.035 & 10.81 & \\
\hline 11 & 4 & 8 & 12 & 3 & 9 & 60529.150 & -0.166 & -83.32 & -0.05 \\
\hline 12 & 2 & 10 & 12 & 3 & 9 & 54403.740 & 0.022 & 2.82 & \\
\hline 11 & 4 & 7 & 12 & 3 & 10 & 38367.350 & -0.133 & -33.60 & -0.05 \\
\hline 11 & 5 & 6 & 12 & 4 & 9 & 15630.540 & 0.253 & -36.46 & -0.05 \\
\hline 13 & 6 & 7 & 12 & 7 & 6 & 30542.580 & -0.250 & -5.36 & 0.07 \\
\hline 13 & 2 & 11 & 13 & 3 & 10 & 56710.020 & -0.088 & -12.10 & \\
\hline 12 & 5 & 8 & 13 & 4 & 9 & 33016.000 & 0.184 & -74.30 & -0.07 \\
\hline
\end{tabular}




\begin{tabular}{|c|c|c|c|c|c|c|c|c|c|}
\hline 12 & 5 & 7 & 13 & 4 & 10 & 27795.180 & 0.073 & -52.71 & -0.07 \\
\hline 14 & 2 & 12 & 14 & 3 & 11 & 61068.050 & 0.027 & -35.36 & \\
\hline 13 & 4 & 9 & 14 & 3 & 12 & 53648.000 & 0.264 & -31.61 & -0.11 \\
\hline 13 & 5 & 8 & 14 & 4 & 11 & 39682.720 & 0.184 & -69.38 & -0.11 \\
\hline 14 & 5 & 9 & 15 & 4 & 12 & 51079.760 & 0.054 & -84.79 & -0.15 \\
\hline 14 & 6 & 8 & 15 & 5 & 11 & 24184.320 & 0.070 & -80.25 & -0.15 \\
\hline 16 & 8 & 9 & 15 & 9 & 6 & 52226.790 & -0.144 & -45.72 & 0.21 \\
\hline 16 & 8 & 8 & 15 & 9 & 7 & 52226.790 & 0.108 & -45.71 & 0.21 \\
\hline 15 & 5 & 10 & 16 & 4 & 13 & 61711.480 & 0.011 & -96.41 & -0.21 \\
\hline 15 & 6 & 10 & 16 & 5 & 11 & 39697.280 & -0.114 & -130.71 & -0.21 \\
\hline 15 & 6 & 9 & 16 & 5 & 12 & 36875.170 & -0.052 & -108.31 & -0.21 \\
\hline 16 & 4 & 12 & 17 & 3 & 15 & 59907.320 & -0.070 & 50.89 & -0.28 \\
\hline 16 & 7 & 10 & 17 & 6 & 11 & 19972.460 & -0.216 & -113.36 & -0.28 \\
\hline 16 & 7 & 9 & 17 & 6 & 12 & 19574.630 & -0.265 & -108.92 & -0.28 \\
\hline 18 & 8 & 11 & 17 & 9 & 8 & 27718.830 & -0.047 & 26.65 & 0.37 \\
\hline 18 & 9 & 10 & 17 & 10 & 7 & 56944.890 & 0.165 & -58.89 & 0.37 \\
\hline 18 & 9 & 9 & 17 & 10 & 8 & 56944.890 & 0.216 & -58.89 & 0.37 \\
\hline 17 & 6 & 11 & 18 & 5 & 14 & 61946.960 & -0.188 & -167.88 & -0.37 \\
\hline 17 & 7 & 11 & 18 & 6 & 12 & 33220.590 & -0.008 & -157.44 & -0.37 \\
\hline 17 & 7 & 10 & 18 & 6 & 13 & 32445.390 & -0.271 & -147.88 & -0.37 \\
\hline 20 & 9 & 12 & 19 & 10 & 9 & 32462.990 & -0.139 & 31.46 & 0.63 \\
\hline 20 & 9 & 11 & 19 & 10 & 10 & 32462.990 & 0.227 & 31.47 & 0.63 \\
\hline 20 & 10 & 11 & 19 & 11 & 8 & 61658.770 & 0.077 & -74.04 & 0.63 \\
\hline 20 & 10 & 10 & 19 & 11 & 9 & 61658.770 & 0.087 & -74.04 & 0.63 \\
\hline 19 & 8 & 12 & 20 & 7 & 13 & 27734.880 & -0.037 & -192.19 & -0.63 \\
\hline 19 & 8 & 11 & 20 & 7 & 14 & 27540.540 & 0.017 & -188.71 & -0.63 \\
\hline 21 & 10 & 12 & 20 & 11 & 9 & 49465.100 & -0.091 & -21.57 & 0.81 \\
\hline 21 & 10 & 11 & 20 & 11 & 10 & 49465.100 & -0.063 & -21.57 & 0.81 \\
\hline 20 & 8 & 13 & 21 & 7 & 14 & 40904.130 & -0.180 & -251.25 & -0.81 \\
\hline 20 & 8 & 12 & 21 & 7 & 15 & 40525.110 & -0.208 & -243.84 & -0.81 \\
\hline 22 & 10 & 13 & 21 & 11 & 10 & 37203.320 & -0.160 & 36.30 & 1.02 \\
\hline 22 & 10 & 12 & 21 & 11 & 11 & 37203.320 & -0.087 & 36.30 & 1.02 \\
\hline 21 & 9 & 13 & 22 & 8 & 14 & 22601.350 & 0.029 & -233.31 & -1.02 \\
\hline 21 & 9 & 12 & 22 & 8 & 15 & 22555.490 & 0.022 & -232.17 & -1.02 \\
\hline 23 & 10 & 14 & 22 & 11 & 11 & 24866.870 & -0.288 & 99.96 & 1.28 \\
\hline 23 & 10 & 13 & 22 & 11 & 12 & 24866.870 & -0.104 & 99.96 & 1.28 \\
\hline 22 & 9 & 13 & 23 & 8 & 16 & 35449.220 & 0.033 & -298.90 & -1.28 \\
\hline 23 & 10 & 14 & 24 & 9 & 15 & 17601.500 & -0.002 & -279.51 & -1.58 \\
\hline 23 & 10 & 13 & 24 & 9 & 16 & 17591.110 & -0.052 & -279.16 & -1.58 \\
\hline 25 & 12 & 14 & 24 & 13 & 11 & 58903.300 & -0.218 & -35.63 & 1.94 \\
\hline 25 & 12 & 13 & 24 & 13 & 12 & 58903.300 & -0.217 & -35.63 & 1.94 \\
\hline 24 & 9 & 15 & 25 & 8 & 18 & 61691.750 & 0.065 & -453.97 & -1.94 \\
\hline 24 & 10 & 15 & 25 & 9 & 16 & 30407.420 & -0.251 & -358.63 & -1.94 \\
\hline 24 & 10 & 14 & 25 & 9 & 17 & 30385.960 & -0.174 & -357.85 & -1.94 \\
\hline 26 & 11 & 16 & 27 & 10 & 17 & 25374.230 & 0.123 & -421.72 & -2.84 \\
\hline 28 & 13 & 16 & 27 & 14 & 13 & 51408.800 & -0.051 & 50.30 & 3.41 \\
\hline 28 & 13 & 15 & 27 & 14 & 14 & 51408.800 & -0.050 & 50.30 & 3.41 \\
\hline 27 & 11 & 17 & 28 & 10 & 18 & 38202.340 & 0.213 & -521.79 & -3.41 \\
\hline 27 & 11 & 16 & 28 & 10 & 19 & 38192.330 & 0.151 & -521.29 & -3.41 \\
\hline 28 & 11 & 18 & 29 & 10 & 19 & 51158.860 & 0.071 & -631.16 & -4.07 \\
\hline 28 & 11 & 17 & 29 & 10 & 20 & 51138.830 & -0.008 & -630.10 & -4.07 \\
\hline 31 & 14 & 18 & 30 & 15 & 15 & 43899.290 & 0.248 & 171.47 & 5.67 \\
\hline 31 & 14 & 17 & 30 & 15 & 16 & 43899.290 & 0.249 & 171.47 & 5.67 \\
\hline 32 & 15 & 18 & 31 & 16 & 15 & 60870.990 & 0.193 & 58.62 & 6.65 \\
\hline 32 & 15 & 17 & 31 & 16 & 16 & 60870.990 & 0.193 & 58.62 & 6.65 \\
\hline 33 & 15 & 19 & 32 & 16 & 16 & 48651.680 & -0.195 & 191.37 & 7.76 \\
\hline 33 & 15 & 18 & 32 & 16 & 17 & 48651.680 & -0.195 & 191.37 & 7.76 \\
\hline
\end{tabular}




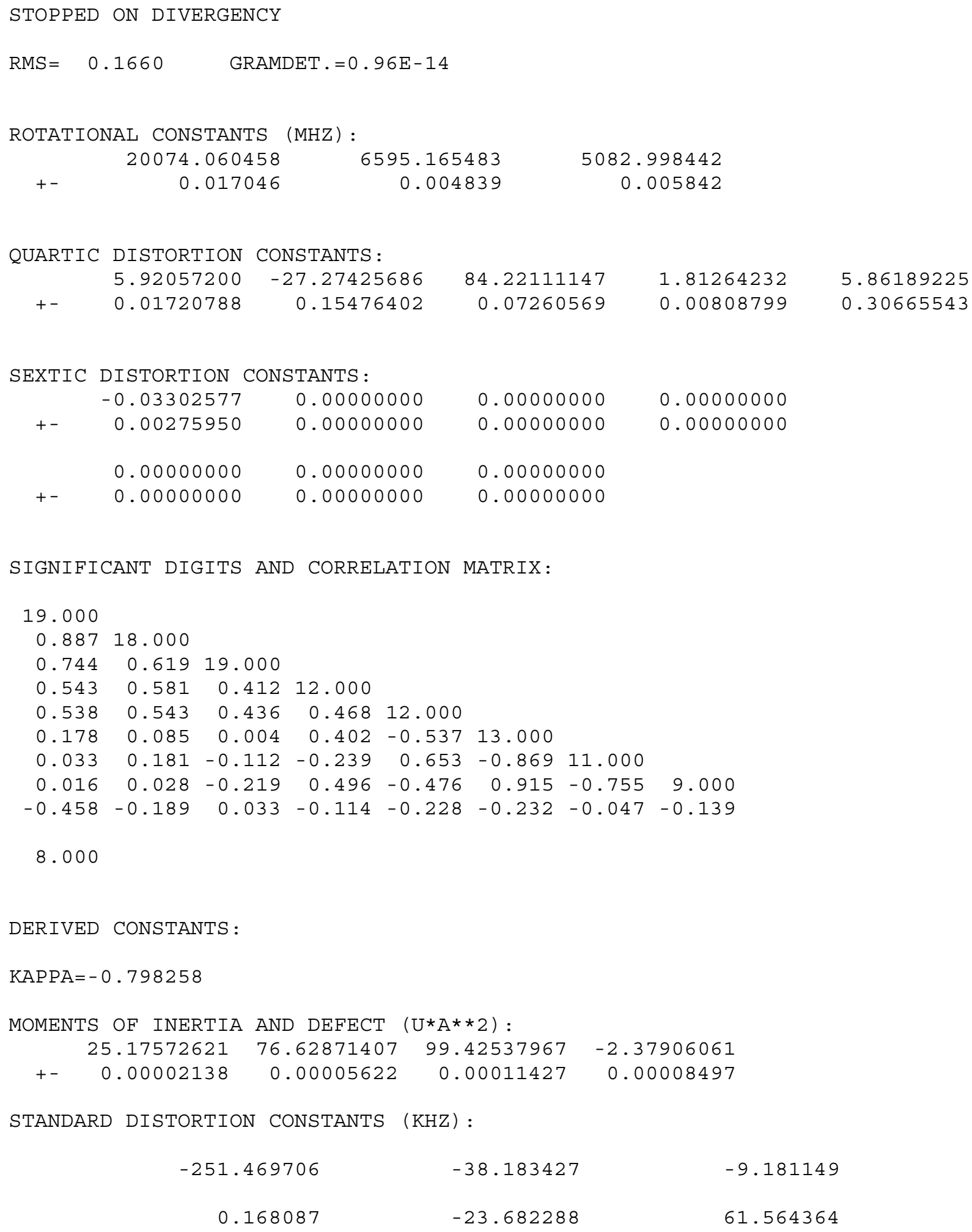

$\begin{array}{rrr}-251.469706 & -38.183427 & -9.181149 \\ 0.168087 & -23.682288 & 61.564364\end{array}$


Table 7S: Microwave Spectrum and Spectroscopic Constants of the Fourth Excited State of the C2-N3 Torsional Vibration of Formic Hydrazide

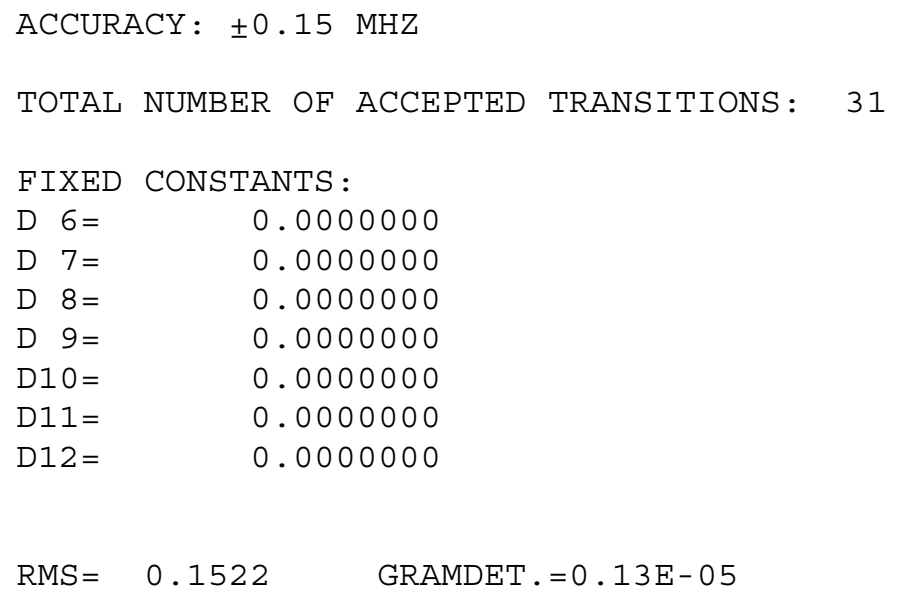

$\begin{array}{rrrrrrrrr}2 & 0 & 2 & 3 & 0 & 3 & 34553.400 & 0.037 & -0.54 \\ 2 & 1 & 1 & 3 & 1 & 2 & 37221.300 & -0.020 & -0.69 \\ 2 & 1 & 2 & 3 & 1 & 3 & 32687.270 & 0.105 & -0.18 \\ 3 & 0 & 3 & 4 & 0 & 4 & 45545.890 & -0.040 & -1.13 \\ 3 & 1 & 2 & 4 & 1 & 3 & 49472.640 & -0.086 & -1.73 \\ 4 & 1 & 3 & 4 & 2 & 2 & 37192.840 & -0.165 & 0.93 \\ 3 & 2 & 2 & 4 & 2 & 3 & 46612.620 & 0.025 & -0.48 \\ 4 & 0 & 4 & 5 & 0 & 5 & 56175.760 & 0.170 & -1.90 \\ 4 & 1 & 3 & 5 & 1 & 4 & 61572.330 & -0.139 & -3.36 \\ 4 & 2 & 3 & 5 & 1 & 4 & 26135.050 & 0.159 & -4.83 \\ 4 & 1 & 4 & 5 & 1 & 5 & 54131.110 & 0.070 & -1.36 \\ 4 & 2 & 2 & 5 & 2 & 3 & 60317.520 & -0.165 & -2.64 \\ 5 & 1 & 4 & 5 & 2 & 3 & 35938.500 & 0.280 & 1.65 \\ 4 & 3 & 1 & 5 & 3 & 2 & 58861.360 & -0.101 & -0.46 \\ 4 & 3 & 2 & 5 & 3 & 3 & 58737.130 & -0.090 & -0.36 \\ 5 & 2 & 4 & 6 & 1 & 5 & 41481.320 & 0.204 & -8.88 \\ 6 & 1 & 5 & 6 & 2 & 4 & 35552.380 & 0.225 & 1.78 \\ 6 & 1 & 6 & 6 & 2 & 5 & 59321.840 & -0.265 & -1.35 \\ 6 & 2 & 5 & 7 & 1 & 6 & 57032.230 & -0.117 & -14.08 \\ 7 & 1 & 6 & 7 & 2 & 5 & 36391.080 & 0.150 & 0.63 \\ 8 & 0 & 8 & 8 & 1 & 7 & 53791.620 & 0.193 & -15.25 \\ 8 & 1 & 7 & 8 & 2 & 6 & 38732.920 & 0.027 & -2.63 \\ 8 & 2 & 6 & 8 & 3 & 5 & 60390.950 & -0.004 & 11.21 \\ 9 & 2 & 7 & 9 & 3 & 6 & 57436.650 & 0.130 & 14.54 \\ 9 & 4 & 6 & 10 & 3 & 7 & 26644.790 & 0.011 & -38.98 \\ 10 & 2 & 8 & 10 & 3 & 7 & 55152.620 & -0.035 & 15.57 \\ 11 & 1 & 10 & 11 & 2 & 9 & 56410.050 & -0.098 & -34.14 \\ 11 & 2 & 9 & 11 & 3 & 8 & 54029.990 & 0.021 & 12.56 \\ 12 & 2 & 10 & 12 & 3 & 9 & 54471.490 & -0.116 & 3.73 \\ 13 & 2 & 11 & 13 & 3 & 10 & 56774.440 & 0.009 & -12.67 \\ 14 & 2 & 12 & 14 & 3 & 11 & 61129.650 & 0.049 & -38.16\end{array}$




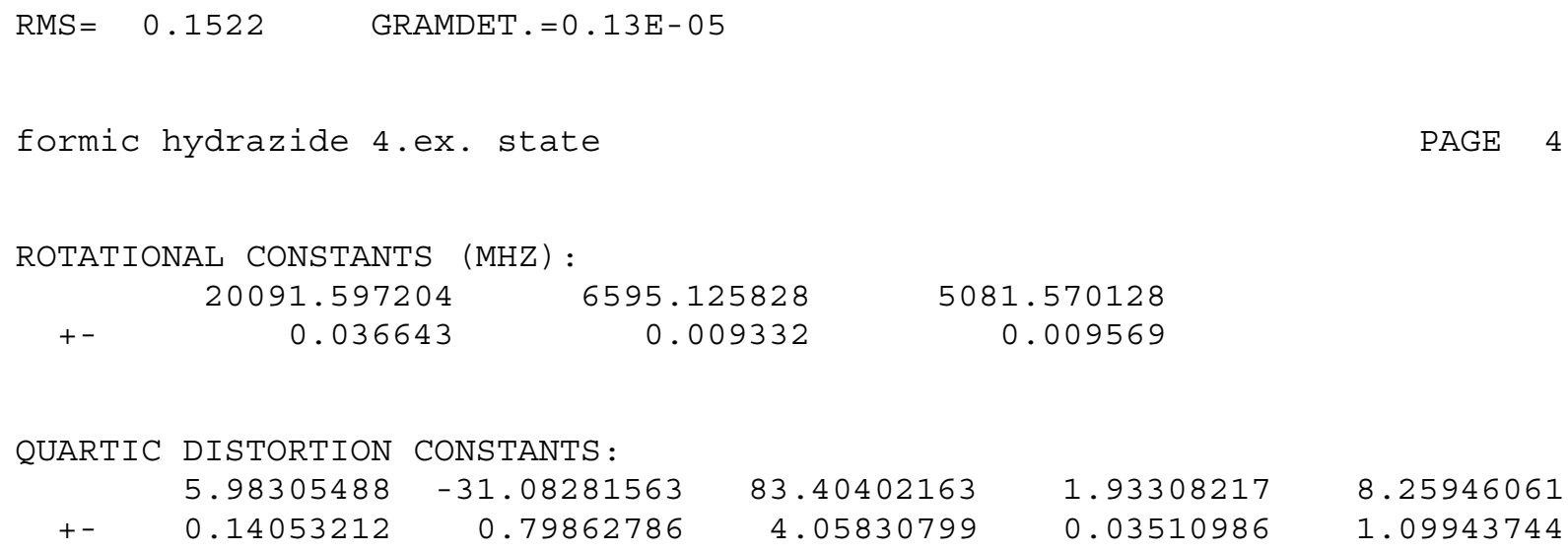

